\title{
Evaluation of ECMWF medium-range ensemble forecasts of precipitation for river basins
}

\author{
J. Ye ${ }^{\mathrm{a}, \mathrm{b} \star}$ Y. He ${ }^{\mathrm{c}}$ F. Pappenberger, ${ }^{\mathrm{d}, \mathrm{a}}$ H. L. Cloke, ${ }^{\mathrm{e}, \mathrm{f}}$ D. Y. Manfull and Z. $\mathrm{Li}^{\mathrm{a}}$ \\ ${ }^{a}$ College of Hydrology and Water Resources, Hohai University, Nanjing, China \\ ${ }^{\mathrm{b}}$ Huai River Basin Meteorological Centre, Bengbu, China \\ ${ }^{c}$ Tyndall Centre for Climate Change Research, School of Environmental Sciences, University of East Anglia, Norwich, UK \\ ${ }^{\mathrm{d}}$ European Centre for Medium-Range Weather Forecasts, Reading, UK \\ ${ }^{e}$ Department of Geography and Environmental Science, University of Reading, UK \\ ${ }^{\mathrm{f}}$ Department of Meteorology, University of Reading, UK \\ ${ }^{\mathrm{g}}$ Institute for Landscape Ecology and Resources Management, University of Giessen, Germany
}

*Correspondence to: J. Ye. E-mail: yejinyin@sina.com

\begin{abstract}
Providing probabilistic forecasts using Ensemble Prediction Systems has become increasingly popular in both the meteorological and hydrological communities. Compared to conventional deterministic forecasts, probabilistic forecasts may provide more reliable forecasts of a few hours to a number of days ahead, and hence are regarded as better tools for taking uncertainties into consideration and hedging against weather risks. It is essential to evaluate performance of raw ensemble forecasts and their potential values in forecasting extreme hydro-meteorological events. This study evaluates ECMWF's mediumrange ensemble forecasts of precipitation over the period 1 January 2008 to 30 September 2012 on a selected midlatitude large-scale river basin, the Huai river basin (ca. $270000 \mathrm{~km}^{2}$ ) in central-east China. The evaluation unit is sub-basin in order to consider forecast performance in a hydrologically relevant way. The study finds that forecast performance varies with sub-basin properties, between flooding and non-flooding seasons, and with the forecast properties of aggregated time steps and lead times. Although the study does not evaluate any hydrological applications of the ensemble precipitation forecasts, its results have direct implications in hydrological forecasts should these ensemble precipitation forecasts be employed in hydrology.

Key Words: $\quad$ ECMWF EPS; Huai; skill score; diurnal cycle

Received 24 March 2013; Revised 4 July 2013; Accepted 16 August 2013; Published online in Wiley Online Library
\end{abstract}

\section{Introduction}

A deterministic weather forecast is a single model trajectory generated by a numerical weather prediction (NWP) system. It is highly dependent on the estimation of the initial atmospheric conditions and does not take uncertainties into consideration. If the initial conditions are incorrect, the forecast will fail to replicate weather events correctly. Since the inherent stochastic nature of a weather system was discussed by Lorenz (1963, 1969), it has been recognised that a perfect numerical weather forecast is unattainable (Hamill et al., 2000) because a tiny error in the initial conditions will grow inevitably and a deterministic forecast is 'determined' to fail. An alternative approach is to incorporate uncertainties by finding reasonable probabilistic distribution functions of atmospheric conditions and generating multiple forecasts from different initial conditions, and sometimes from different model parametrizations. Leith (1974) called such forecasts 'Monte Carlo Forecasts', now usually referred to as an Ensemble Prediction System (EPS). Leith (1974) suggests the mean of a forecast ensemble is the estimate of the true state of the atmosphere that is best in the least-square-error sense. Buizza (2008) interprets EPS as a system based on a finite number of deterministic integrations and the only feasible method in meteorology to predict a probability density function beyond the range of linear error growth. EPS has been embraced by the meteorological community as a practical way of estimating uncertainties of a weather forecast (Hamill et al., 2000). It has also become popular in the field of hydrology and water resources management (Thielen et al., 2008), which has been demonstrated by the Hydrologic Ensemble Prediction EXperiment (HEPEX) (for a comprehensive review see Cloke and Pappenberger (2009) and visit www.hepex.org) for a wide range of water-related hazards (Alfieri et al., 2012).

The performance of EPS has been constantly assessed by meteorologists and more recently by hydrologists using a variety of ensemble verification statistics such as the Brier score (BS), the ranked probability score (RPS), the relative operating characteristic (ROC) and others (see e.g. Cloke and Pappenberger, 2008). Meteorologists usually compute the statistics of variables that may not be directly relevant for hydrology, e.g. geopotential 
1 height at $500 \mathrm{hPa}$ (Molteni et al., 1996), or may not have the appropriate spatial scales for hydrological models, e.g. average precipitation over grids (Buizza, 1999). Readers can also see Buizza (2009). It is therefore difficult to draw conclusions on the efficacy of EPS when applied in the field of hydrology and other associated fields resulting often in the development of novel scores reflecting the needs of a particular community (Pappenberger et al., 2011a). Most studies of hydro-meteorological forecast systems include not only an evaluation of forecast hydrological 0 forecast skill but also meteorological skill (e.g. Pappenberger 11 et al., 2005, 2011b; De Roo et al., 2011; Voisin et al., 2011). There 12 are a number of studies that assess the 'hydrological' quality 13 of EPS by focusing on the performance of ensemble forecasts of 14 precipitation and the simulated ensemble discharge. For example, 15 Thirel et al.(2008) assess the quality of the European Centre for 6 Medium-range Weather Forecasts (ECMWF) and Météo-France 6 Prévision d'Ensemble ARPEGE (PEARP) EPS precipitation over 17 France using the Brier skill score (BSS) and the ranked probability 18 skill score (RPSS). Velázquez et al.(2009) used Continuous 19 Ranked Probability Score (CRPS) and the rank histogram for 20 evaluating a Canadian hydrological ensemble prediction system 21 (H-EPS). He et al.(2009) evaluated the performance of ensemble 22 precipitation and discharge forecasts of January 2008 from seven 23 forecast centres for the Upper Severn catchment using CRPS 24 and ROC. Such studies are valuable in facilitating hydrological 25 applications of EPS. But they do not necessarily provide detailed analysis of the ensemble precipitation, e.g. the performance at different time steps (in particular sub-daily time steps), lead times or for river basins with various properties, and most of them only study a number of individual events or seasons.

Ensemble forecasts have yet to be used to their full potential, 30 although they have been produced for nearly two decades. One of 31 the main reasons is that their performance is often deemed 32 to be too poor to provide 'harmless' operational forecasts. 33 Their uncertainties are considerably large especially as the lead 34 times increase. False alarms do not only cost significantly in 35 financial terms but also damage the reputation of forecasting 36 institutions. Operational forecasters often have to make a binary 7 decision whether or not an action should be taken. It is not so straightforward for decision makers to utilise ensemble forecasts 8 in terms of probabilities compared to conventional deterministic 39 forecasts whereby a binary decision can be made based on a single 40 forecast, albeit with inevitable errors in the single forecast. This is 41 often the second 'excuse' for ignoring ensemble forecasts. Readers 42 can refer to Demeritt et al. (2010) for more detailed discussion on 43 challenges in communicating and using ensembles in operational 44 flood forecasting. This article aims to address the first question: 45 how poor or how good the forecasts are, based on the current 6 generation of model and data assimilation methods.

ECMWF has been producing short to medium range (0-15 days forecast lead time) ensemble weather forecasts operationally since November 1992, Such weather forecasts have 49 also been produced at a number of other centres, along with 50 ECMWF, which have recently shared products and emerged 51 into the so-called TIGGE initiative, acronym for THORPEX 52 Interactive Grand Global Ensemble (Bougeault et al., 2010), which 53 has been used in many hydrometeorological forecasting studies 54 (e.g. Pappenberger et al., 2008b; He et al., 2009). This article an be applied to study other ensemble prediction systems.

The Huai river basin is selected in this study because it has a good-quality precipitation observational dataset. It is located in midlatitudes straddling the southern monsoon and the northern continental climate, which makes the basin an interesting and challenging test bed. The basin encompasses one of the fastest growing economic regions in China but is highly vulnerable to extreme hydrometeorological events, with floods as the worst disaster in this basin. Its average population density is $c a .600 \mathrm{~km}^{-2}$ (Ning et al., 2003), more than four times the national average of discussion in Pappenberger et al.(2008a) and Pappenberger and

$138 \mathrm{~km}^{-2}$. Major basin-wide floods have been recorded once every 5 years on the average and local floods once every 2 or 3 years (Huai River Commission, Ministry of Water Resources, 2010) and affect millions of people. The period between May and September is officially regarded as the basin's flooding season, although large spring floods have occurred in April a number of times in past years. Snowfall is rare and thus large floods are mainly driven by heavy rainfall. Due to the significant economic value of the basin and its frequent devastating floods, a number of recent studies have pointed to the potential of using ensemble forecasts in this basin. He et al.(2010) use six forecast centres from the TIGGE archive to drive a coupled atmospheric-hydrologic cascade system to hindcast three 2007 flood events on the Upper Huai sub-basin $\left(30672 \mathrm{~km}^{2}\right.$ ). The results demonstrate that the TIGGE multi-model ensemble has great potential to produce skilful forecasts of river discharge and improve the warning time to as early as 10 days in advance. Yang et al.(2012) use generalised additive models and Bayesian model averaging (BMA) to post-process the ensemble forecasts from the National Centers for Environmental Prediction (NCEP).The method was applied to the Yishusi river sub-basins, in the eastern part of the Huai river basin, for July 2007. The BMA forecasts outperform the raw ensemble forecasts especially for extreme precipitation. Liu et al.(2013) evaluate the forecasting skills of post-processed ensemble forecasts from a fixed version of the Global Forecast System (GFS) produced by NCEP. Their study is carried out for 15 sub-areas of the Huai river basin for 23 years starting from 1981. The post-processing method applied in their study can remove all the biases in the raw ensemble forecasts, and improve the forecasting skill and ensemble spread.

The above-mentioned studies however did not carry out detailed analysis of the performance of the raw ensemble forecasts which is the basis of both precipitation post-processing and further application in hydrology and other fields. This was partly due to the fact that a homogenised precipitation database for the entire basin was not readily available to enable a rigorous performance evaluation of the raw forecasts. Bröcker (2012) points out that performance evaluation of raw ensembles may serve as a benchmark for more sophisticated ensemble interpretation models. The availability of hourly observed precipitation analysis at high spatial resolution produced by the China Meteorological Administration (CMA) has provided a perfect opportunity to evaluate ensemble forecasts near real time and at sub-daily resolutions.

This article aims to carry out an in-depth assessment of ECMWF's medium-range ensemble precipitation forecasts and address three scientific questions: (i) how skilful are ECMWF's ensemble forecasts over this midlatitude river basin; (ii) how do the forecast skills vary with seasons, sub-basin properties, lead times and aggregated time steps; and (iii) do sub-daily ensemble forecasts bring any benefits or are they simply unwanted noise generated by the current model version? The article is organised in the following way. The study area and data are described in section 2. The experimental design and scores used for evaluation are explained in section 3. The results are presented and discussed in section 4 , which is followed by the final concluding section.

\section{Study area and data description}

\subsection{The Huai river basin}

The Huai river basin is located in central east China, between the lower reaches of the Yellow and the Changjiang (Yangtze) Rivers $\left(112-121^{\circ} \mathrm{N}, 31-36^{\circ} \mathrm{E}\right)$. It has a total drainage area of approximately $270000 \mathrm{~km}^{2}$ (Figure 1). It consists of two river systems, namely the Huai rivers and the Yishusi rivers. The Huai originates from the Tongbai Mountains in Henan province, flows towards the east through Henan, Anhui and Jiangsu provinces. The total length of the Huai's main reach is over $1000 \mathrm{~km}$ with

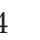
. 6 7 9 


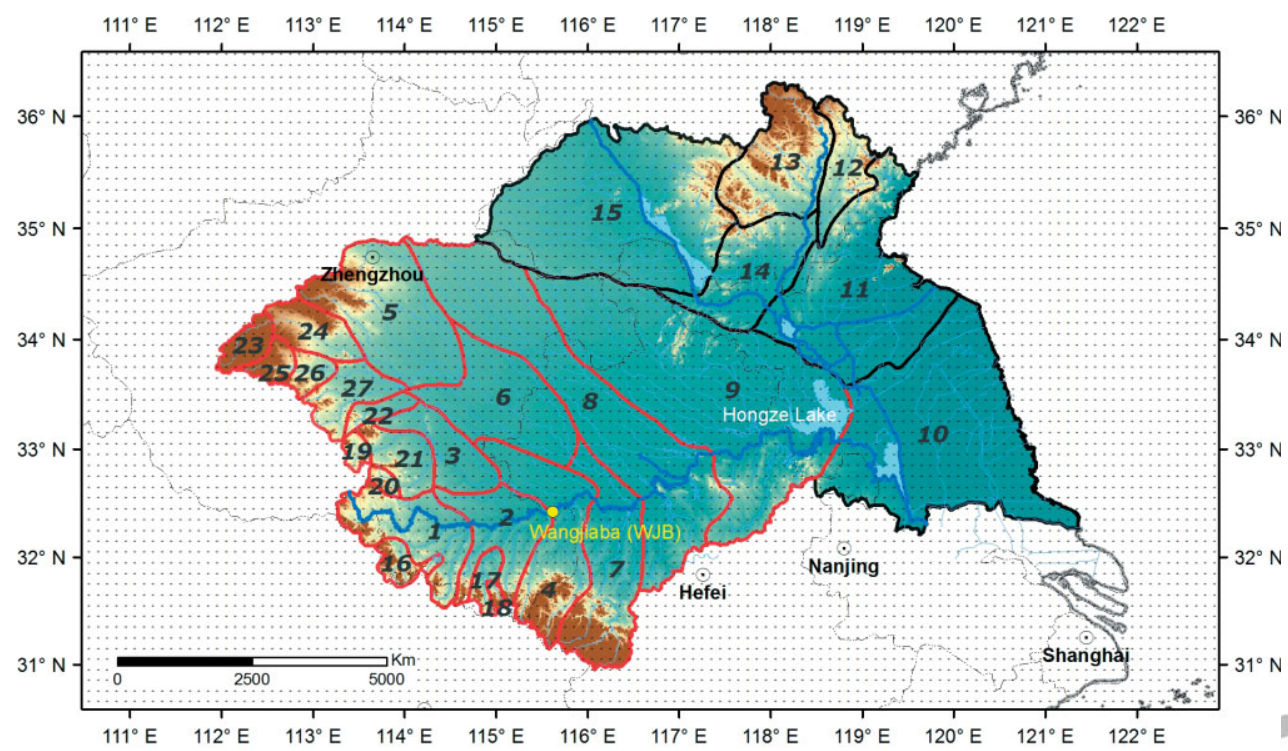

Legend

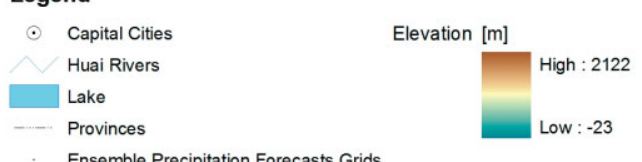

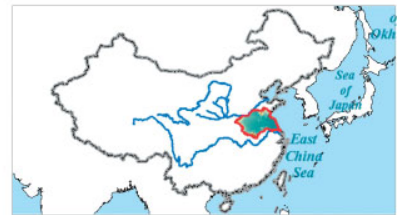

Figure 1. Elevation map of 27 Huai sub-basins (refer to Table 1 for their characteristics) located in central east China. The location is indicated in the right lower panel with blue lines representing the Yellow and Changjiang rivers. Sub-basins nos. 10-15 belong to the Yishusi rivers and the remaining belong to the Huai rivers.

an average elevation drop of $200 \mathrm{~m}$, of which $178 \mathrm{~m}$ of the drop is up to the Wangjiaba (WJB) sluice gate, $22 \mathrm{~m}$ up to Hongze Lake. The relatively low elevation drop in the middle and lower reaches $(22 \mathrm{~m})$ leads to flattened flow velocity and increased risk of over-bank flow and inundation. To the northeast side of the basin is the Yishusi river system, most of which is on the former Yellow river flood plain as a product of ancient Yellow river floods and resulting river path alterations. The Yishusi originates from the Yimeng Mountains in Shandong province and mostly flows through Shandong and Jiangsu provinces and joins the Hongze Lake in the south. It has over 15 tributaries or channels that empty into the Yellow Sea. The entire basin is divided into 27 sub-basins, merging two sub-basin boundary definitions used by the Huai Basin Meteorological Centre and the Huai River Commission. Sub-basins nos. 10-15 belong to the Yishusi and the remaining to the Huai. Due to thousands of existing hydraulic structures in the basin, its sub-basin delineation cannot solely depend on the natural topography and elevation. Table 1 lists characteristics including area, centroid coordinates, mean elevation, mean annual precipitation, mean annual temperature, and reliability of the precipitation time series used in the analysis in the form of correlation $R^{2}$. The last column will be explained in section 4.1 .

The mean annual precipitation and runoff depth of the entire Huai river basin is approximately 888 and $230 \mathrm{~mm}$ respectively. The precipitation dynamics including spatial and temporal distribution is very irregular and changes from year to year. This is attributed to the basin location in the transitional area between the southern monsoon and the northern continental climate (Huai River Commission, Ministry of Water Resources, 1999). The East Asian summer monsoon rainfall (known as Meiyu in China) usually occurs during June and early July over the basin. The onset, duration and total rainfall amount of the Meiyu season usually determine the severity of the basin's annual floods. The Meiyu front generates widespread, persistent and heavy rainfall over the basin $(\mathrm{Fu}, 1991)$. Occasional remnants of typhoons may affect the basin from late July to September and can cause the most intense precipitation (Fu, 1991; Svensson and Rakhecha, 1998) although the distance of the far western point of the basin to the sea is over $900 \mathrm{~km}$. For example, an intensive storm event recorded at the Linzhuang station (located in sub-basin no. 21) reached $830 \mathrm{~mm}$ within just $6 \mathrm{~h}$ and was caused by typhoon Nina (Bao, 1987). The areal extent of typhoon rainfall is smaller and duration is shorter compared to those of the Meiyu front (Fu, 1991; Svensson and Rakhecha, 1998). A storm event is defined in China as $24 \mathrm{~h}$ rainfall larger than $50 \mathrm{~mm}$ and smaller than $100 \mathrm{~mm}(50 \leq 24 \mathrm{~h} P<100)$. The flooding season in China is defined as the period between May and September inclusive. Cheng (2004) reveals that between June and August the basin has on average 2.3 days of storm events, the largest 5.1 days of storms in 1954 being one of the wettest years, and the lowest 0.7 day of storms in 1966 being one of the driest years. In the flooding season, stratiform-cloud rain and convective rain are commonly seen in the Huai river basin. The latter causes the intensive and local events. Larger extent but intensive rains can be cumulus-stratus mixed precipitation. Stratiform-cloud rain usually dominates in the non-flooding season.

\subsection{Observed precipitation analysis}

The observed precipitation dataset was obtained from the Climatic Data Centre (CDC), National Meteorological Information Centre, China Meteorological Administration (CMA). It combines two sources of precipitation data, namely ground-based rain-gauges and satellite-based precipitation. The ground-based rain-gauges consist of over 30000 automatic observation stations, including national and regional automatic weather stations which record precipitation at an hourly time step. It is worth noting here that only hourly rain-gauge data were used to produce this dataset. The satellite-based precipitation is the global precipitation product created by the National Oceanic and Atmospheric Administration Climate Prediction Center (NOAA CPC) Morphing Technique (CMORPH) (Joyce et al., 2004) that is derived from low-orbiter satellite microwave observations exclusively. It has a spatial resolution of $0.07277^{\circ} \times 0.07277^{\circ}$ and temporal resolution of $30 \mathrm{~min}$.

CMA's hourly rain-gauge data are first spatially interpolated to $0.1^{\circ} \times 0.1^{\circ}$ latitude/longitude grids. The CMORPH data are resampled to the same $0.1^{\circ} \times 0.1^{\circ}$ grids and hourly time steps, and then corrected against the rain-gauge data using a Probability 


\begin{tabular}{|c|c|c|c|c|c|c|}
\hline$\overline{\text { ID }}$ & Area $\left(\mathrm{km}^{2}\right)$ & $\begin{array}{c}\text { Centroid coordinates } \\
\text { (longitude/latitude in degrees) }\end{array}$ & $\begin{array}{l}\text { Mean elevation } \\
\quad(\mathrm{m} \text { a.s.l. })\end{array}$ & $\begin{array}{l}\text { Mean annual } \\
\mathrm{P}(\mathrm{mm})\end{array}$ & $\begin{array}{l}\text { Mean annual } \\
\mathrm{T}\left({ }^{\circ} \mathrm{C}\right)\end{array}$ & $\begin{array}{c}\mathrm{R}^{2}(\mathrm{CMORPH}-\text { Gauge } \\
\text { merged vs. Gauge) }\end{array}$ \\
\hline 1 & 8382 & $114.09 / 32.23$ & 139 & 1049 & 15.1 & 0.8744 \\
\hline 2 & 7167 & $115.03 / 32.22$ & 75 & 1035 & 15.2 & 0.8128 \\
\hline 3 & 5017 & $114.46 / 32.98$ & 52 & 914 & 14.9 & 0.8978 \\
\hline 4 & 11407 & $115.57 / 32.20$ & 141 & 1074 & 15.3 & 0.9047 \\
\hline 5 & 14242 & $113.85 / 34.23$ & 134 & 696 & 14.5 & 0.9080 \\
\hline 6 & 11566 & $115.09 / 33.42$ & 41 & 815 & 14.8 & 0.9204 \\
\hline 7 & 12115 & $116.21 / 31.90$ & 205 & 1126 & 15.4 & 0.9009 \\
\hline 8 & 28498 & $115.96 / 33.43$ & 44 & 825 & 14.8 & 0.9569 \\
\hline 9 & 40089 & $117.31 / 33.54$ & 37 & 825 & 14.1 & 0.9623 \\
\hline 10 & 32162 & $119.84 / 33.14$ & 6 & 851 & 12.3 & 0.9679 \\
\hline 11 & 21472 & $118.96 / 34.44$ & 31 & 795 & 12.5 & 0.9773 \\
\hline 12 & 4282 & $118.78 / 35.55$ & 167 & 782 & 12.8 & 0.9293 \\
\hline 13 & 10185 & $118.10 / 35.60$ & 248 & 756 & 13.1 & 0.9403 \\
\hline 14 & 9240 & $117.80 / 34.63$ & 64 & 808 & 13.9 & 0.9682 \\
\hline 15 & 31148 & $116.35 / 35.17$ & 66 & 666 & 13.9 & 0.9789 \\
\hline 16 & 1769 & $113.97 / 31.95$ & 257 & 1073 & 15.1 & 0.7870 \\
\hline 17 & 1381 & $114.90 / 31.80$ & 142 & 1163 & 14.7 & 0.8155 \\
\hline 18 & 333 & $114.99 / 31.65$ & 267 & 1213 & 14.5 & 0.7427 \\
\hline 19 & 815 & $113.47 / 32.99$ & 213 & 917 & 14.7 & 0.7255 \\
\hline 20 & 635 & $113.78 / 32.71$ & 224 & 976 & 14.9 & 0.7317 \\
\hline 21 & 3672 & $113.97 / 32.92$ & 122 & 945 & 14.8 & 0.9154 \\
\hline 22 & 1387 & $113.71 / 33.36$ & 103 & 876 & 14.7 & 0.8802 \\
\hline 23 & 1941 & $112.29 / 33.95$ & 796 & 697 & 14.4 & 0.7198 \\
\hline 24 & 3760 & $113.01 / 34.08$ & 264 & 693 & 14.4 & 0.9050 \\
\hline 25 & 1436 & $112.57 / 33.79$ & 497 & 741 & 14.6 & 0.8059 \\
\hline 26 & 1539 & $112.96 / 33.70$ & 192 & 780 & 14.7 & 0.8950 \\
\hline 27 & 3978 & $113.39 / 33.50$ & 131 & 815 & 14.6 & 0.8992 \\
\hline
\end{tabular}

61 The observed precipitation analysis product, the CMORPH62 Gauge merged data, was accumulated to daily time steps and 63 evaluated against the data collected from the basin's daily 64 rain-gauges which are independent from the hourly gauges used

in the merged dataset. Because the daily rain-gauges record daily precipitation data from Beijing time (UTC $+8 \mathrm{~h}) 2100$ to Day $+1 / 2100$, the CMORPH-Gauge merged data is accumulated from 1300 UTC to Day + 11300 UTC to be consistent with the rain-gauges. Unlike the hourly rain-gauges used in the CMORPHGauge merged data, the data collected from the daily rain-gauge network was quality controlled and contains a larger number of gauges than that of the hourly rain-gauges. The daily raingauge data are therefore considered as a reasonable benchmark to cross-check the quality of the CMORPH-Gauge merged data. The correlation between CMORPH-Gauge merged data and daily rain-gauge data was computed for each sub-basin over the entire study time period.

After the quality of the precipitation data was examined, the forecast performance was evaluated. The entire study time period was divided into ten segments, composed of five flooding and five non-flooding seasons. The flooding season covers five months, namely May, June, July, August and September. The non-flooding season covers seven months, namely October, November, December, January, February, March and April. Except for the first segment, the 2008 non-flooding season that covers four months (1 January 2008 to 30 April 2008), the remaining nine segments all span the entire season. The flooding and non-flooding seasons alternate and end with the 2012 flooding season (1 May 2012 to 30 September 2012). The ECMWF ensemble precipitation forecasts were evaluated for all ten seasons, 27 sub-basins, at five different aggregated time steps, namely 3, 6, 12, 24 and $48 \mathrm{~h}$, and all the available lead times.

\subsection{Evaluation scores}

The Continuous Ranked Probability Score (CRPS:Brown, 1974; Matheson and Winkler, 1976; Hersbach, 2000) and two variants of CRPS were used as evaluation scores. The CRPS is a verification tool that evaluates the degree of agreement between the cumulative probability distribution of an ensemble of variable values with a single observed value.

$$
\text { CRPS }=\int_{-\infty}^{\infty}\left\{P(x)-H\left(x-x_{\mathrm{a}}\right)\right\}^{2} \mathrm{~d} x
$$


where $x$ is the forecasted variable, $x_{\mathrm{a}}$ is the actual variable value (the observed value), $P(x)$ is the cumulative distribution function of $x$, and $H\left(x-x_{\mathrm{a}}\right)$ is the Heaviside function which is 0 when $\left(x-x_{\mathrm{a}}\right)<0$ and 1 otherwise. The unit of CRPS is the same as that of $x$. The ideal degree of agreement $(C R P S=0)$ is achieved if $P(x)=H\left(x-x_{\mathrm{a}}\right)$, which is a perfect deterministic forecast. In practice, CRPS usually takes the average value over an area and a number of forecasting cases. This potentially leads to a technical problem when scores need to be compared amongst different areas, seasons or aggregated time steps when the $x$ values can assume various magnitudes. In other words, a lower CRPS in a particular area or season does not necessarily equate to a better forecasting performance in comparison with another area or season. This is because a lower score can be attributed to lower $x$ values but not a better forecast over a certain area or season. RCRPS, a normalised CRPS, was introduced by Trinh et al.(2013) to handle this technical problem. It normalises CRPS by the standard deviation of the variable of interest.

$$
R C R P S=\frac{C R P S}{\sigma_{\mathrm{a}}}
$$

where $\sigma_{\mathrm{a}}$ is the standard deviation of all $x_{\mathrm{a}}$ values over a certain area and a number of studied cases. Another normalised form of CRPS is the Continuous Ranked Probability Skill Score (CRPSS), where CRPS is normalised by a reference which is usually the climatology or persistence of a study area.

$$
C R P S S=1-\frac{C R P S_{\mathrm{F}}}{C_{R} P S_{\mathrm{R}}}
$$

where $C R P S_{\mathrm{F}}$ denotes the forecast score and $C R P S_{\mathrm{R}}$ is the score of a reference forecast of the same variable. CRPSS measures the improvement of an ensemble forecasting system over the reference forecast. Its values range from $-\infty$ to 1 , where 1 is the ideal forecast and negative values indicate worse performance than the reference forecast. The reference forecast used in this study is climatology, which was computed for flooding or non-flooding seasons respectively over each sub-basin and each accumulated time step using the observed precipitation analysis data from January 2008 to 10 October 2012.
The dependency of CRPSS on sub-basin properties is studied 1 using a multiple regression function. CRPSS is based on $24 \mathrm{~h} 2$ aggregated precipitation and averaged over the five flooding and 3 five non-flooding seasons respectively.

$$
\text { CRPSS }=a+b_{1} C S+b_{2} M E+b_{3} M A P,
$$

where $a$ is the intercept, $b_{i}$ is the coefficient for each variable, CS is the sub-basin size, $M E$ is the mean elevation, and MAP is the mean annual precipitation. The variables CS, ME and MAP are all normalised by the maximum of each variable.

\section{Results and discussion}

\subsection{Quality of observed precipitation data}

The CMORPH-Gauge merged precipitation dataset is a product based on hourly rain-gauge and satellite data. Its quality was checked against the daily rain-gauge data which is considered as a benchmark. The correlations between the merged data accumulated to 24 hourly (on the $x$-axis) and the daily rain-gauge data (on the $y$-axis) over the time period between 1 January 2008 and 31 December 2011 were obtained for the 27 sub-basins using linear regression functions (figures are not shown here). The least square errors $R^{2}$ were computed (last column in Table 1). The correlations are generally better for larger sub-basins and sub-basins with lower elevations (Figure 2), and vice versa. The $y$-intercepts obtained for all the 27 linear regression functions are positive, indicating underestimation of precipitation in the CMORPH-Gauge merged data in comparison with the daily rain-gauge data. Xie et al.(2007) reports a similar finding that the CMORPH product underestimates the precipitation amount over eastern China. The sub-basins with the largest and smallest $R^{2}$ are sub-basin no. 15 with an area of $31148 \mathrm{~km}^{2}$ and mean elevation of $66 \mathrm{~m}$, and sub-basin no. 23 with an area of $1941 \mathrm{~km}^{2}$ and mean elevation of $796 \mathrm{~m}$ (Figure 3).

The advantage of using the merged data lies in its high spatial and temporal resolutions which make it possible to evaluate forecast performance at sub-daily time-scales. The correlations for the 27 sub-basins are all above 0.7 and acceptable. Nevertheless, the uncertainties associated with the skill scores caused by the limitation in the high spatial and temporal resolution precipitation analysis product need to be recognised.

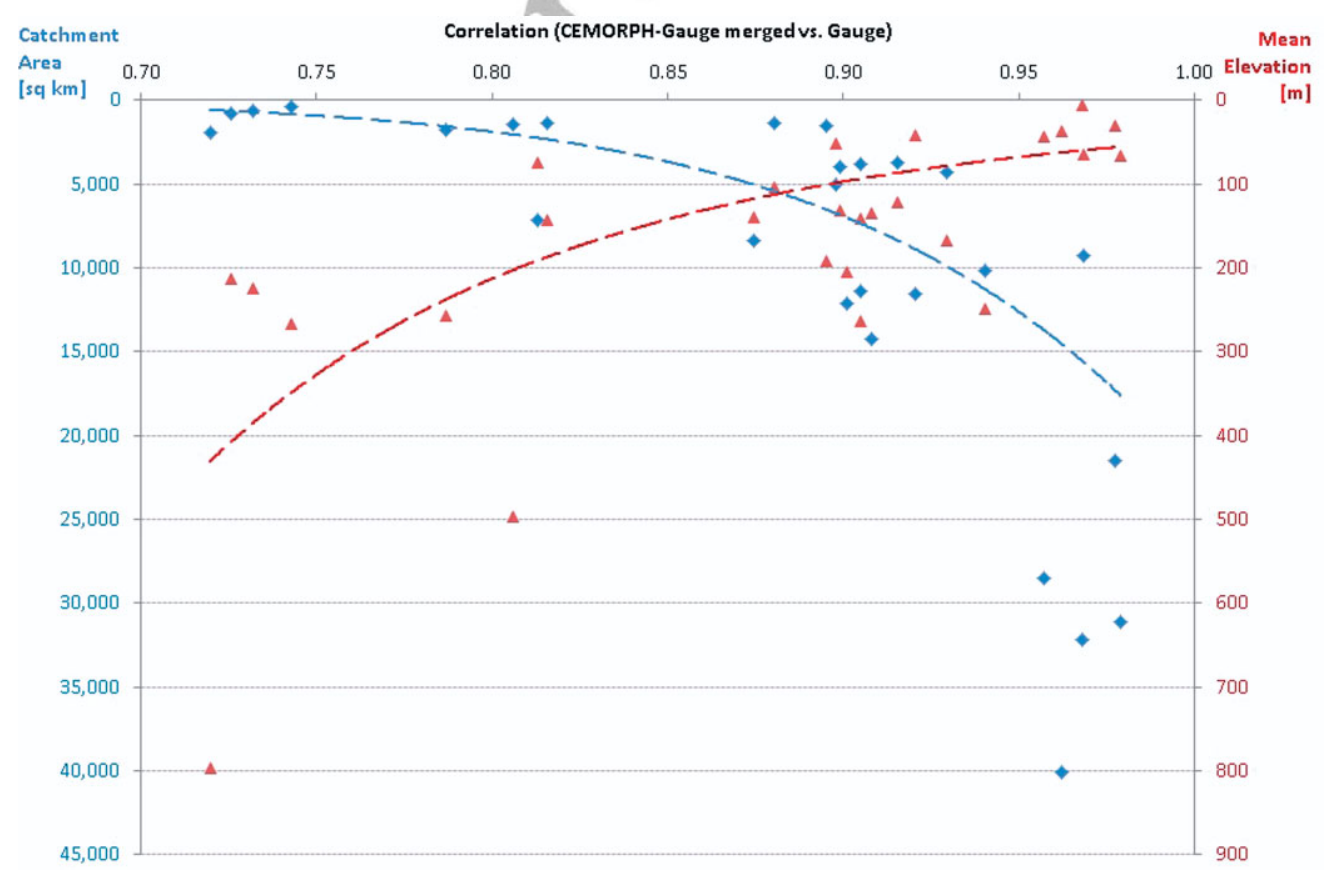

Figure 2. The relationship between sub-basin areas (left axis, diamond markers)/mean elevation (right axis, triangular markers) and correlations $\mathrm{R}^{2}$ (between CMORPH-Gauge merged and rain-gauge precipitation time series at $24 \mathrm{~h}$ intervals). Correlations are higher for larger sub-basins and sub-basins dominated by flat terrains (refer to the last column in Table 1 for the correlation $\mathrm{R}^{2}$ ). This figure is available in colour online at wileyonlinelibrary.com/journal/qj 


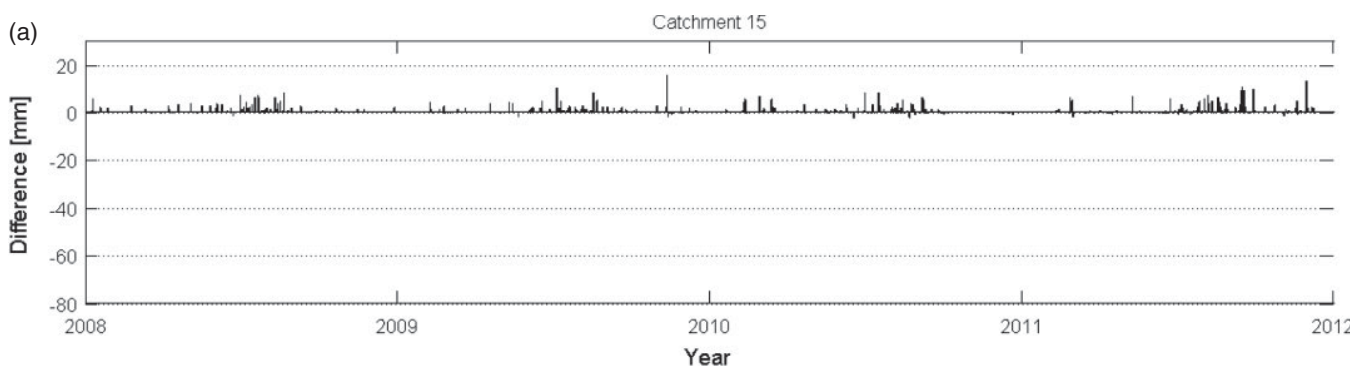

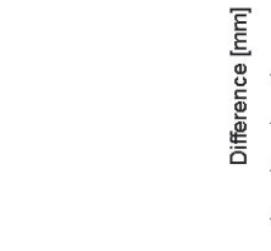

(b) Catchment 23

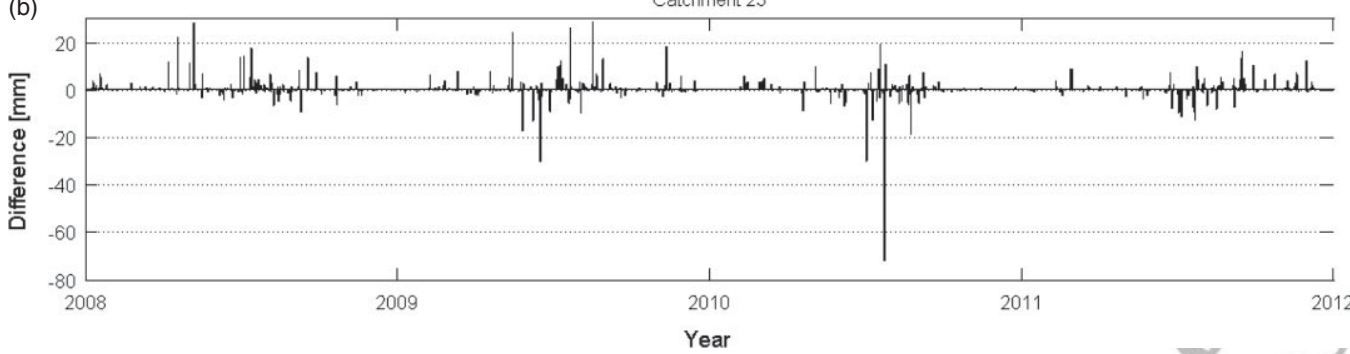

Figure 3. (a), (b) The difference in the daily precipitation time series (rain gauge - CMORPH-Gauge merged) for the best and worst correlated sub-basins nos. 15 and 23 respectively. In the majority of cases the CMORPH-Gauge merged data underestimate the daily precipitation values. The precipitation data in 2012 were not evaluated because the quality control of the daily rain-gauge data would not be completed until the first quarter of 2013 .

\subsection{The overall skill scores of ECMWF's ensemble forecasts}

CRPSS is used to evaluate the overall performance of ECMWF's ensemble forecasts in comparison with climatology. Figure 4 shows CRPSS calculated using $24 \mathrm{~h}$ accumulated precipitation and averaged over the five flooding and five non-flooding seasons respectively. Sub-plots are presented in ascending order of sub-basin size. All sub-basins, except nos. 5, 12, 13, 23, 24 and 25, in both flooding and non-flooding seasons show overall decreasing skill scores with increasing lead times for the forecasted precipitation. The six atypical sub-basins exhibit rising or fluctuating skill scores with increasing lead times. Sub-basins nos. 5, 23, 24 and 25 are located towards the north-west of the basin dominated by the Tongbai mountains, and sub-basins nos. 12 and 13 are located towards the north-east dominated by the Yimeng mountain ranges. The ensemble forecasts completely failed to show any skill in these six sub-basins all located between $34^{\circ} \mathrm{N}$ and $36^{\circ} \mathrm{N}$ and characterised by high altitudes. This may suggest the need for local models in the areas dominated by high altitude to resolve rain-driven processes at small scales.

The skill scores vary depending on the seasons and the sizes of the sub-basins. Flooding seasons (line with dots) showed higher skill scores than non-flooding seasons (line with circles). This may indicate it is easier to correctly forecast rain occurrence and magnitude in a wet season than in a dry season, and the forecasts tend to be more skilful in the wet season compared to the dry season. CRPSS for the flooding seasons never drops below 0 , which means the forecasted precipitation for all 27 sub-basins was more skilful than their climatology. For sub-basins smaller than $2000 \mathrm{~km}^{2}$ (the first row of sub-basins in Figure 4) except nos. 23 and 25, the highest scores never exceeded 0.4 during flooding seasons. The scores are much lower during the non-flooding seasons and most of them show no skill at all $(C R P S S<0)$. For the sub-basins smaller than $10000 \mathrm{~km}^{2}$ (the second row of subbasins in Figure 4) except nos. 12, 13 and 24, the scores appear to be better than the first row of sub-basins. The best scores were achieved by the sub-basins larger than $10000 \mathrm{~km}^{2}$ (the last row of sub-basins in Figure 4) except no. 5. In general, the forecast skill improves as the sub-basin size increases, and forecasts in the flooding seasons outperform those in the non-flooding seasons.

For midlatitude sub-basins like the ones in the Huai river basin, ECMWF's ensemble forecasts can be used in forecasting floods with relatively low, medium and high confidence during flooding seasons for sub-basins with sizes $<2000,2000-10000$, $>10000 \mathrm{~km}^{2}$ respectively. The exception here is the sub-basin dominated by high elevations. During non-flooding seasons, ECMWF's ensemble forecasts did not show satisfactory skills for sub-basins smaller than $2000 \mathrm{~km}^{2}$, but some reasonable skill for sub-basins larger than $2000 \mathrm{~km}^{2}$. Overall, the forecasts are more skilful in the flooding seasons than the non-flooding seasons over this midlatitude river basin.

\subsection{Skill dependency on sub-basin properties and seasons}

The dependency of CRPSS on sub-basin properties is further studied using the multiple regression function (Eq. (4) in section 3.2). CRPSS is based on $24 \mathrm{~h}$ accumulated precipitation and averaged over the five flooding and five non-flooding seasons respectively, the same as what was used in Figure 4.

Sub-basins nos. 5, 12, 13, 23, 24 and 25 were excluded from finding the multiple regression function due to their unusual pattern of CRPSS already discussed in section 4.2. In addition, sub-basins nos. 4 and 7 were also excluded because they do not exhibit homogeneous sub-basin properties with respect to elevation and may interfere with the results. The two sub-basins in question have a large portion of high elevation towards the south side but are fairly flat towards the north. After excluding the seven sub-basins, the obtained regression function based on the remaining 20 sub-basins for the flooding season is given in Table 2.

For the flooding seasons, the coefficients of determination $R^{2}$ of all ten lead times are no less than 0.655 (lowest value appeared on day 5), which indicates that the obtained regression can account for at least $65.5 \%$ of the original variability and the regression model fit is satisfactory. Figure 5 shows an example of the fitted CRPSS versus the original CRPSS for the flooding season on day 2. For flooding seasons, the coefficients of CS $\left(b_{1}\right)$ for the ten lead times are all positive, implying CRPSS increases with increase of $C S$. The coefficients of $M E\left(b_{3}\right)$ are mostly negative (except days 3-5) and close to 0 . This means $M E$ has a relatively smaller influence on CRPSS than CS and MAP have during flooding seasons. In most cases the higher the mean elevation of a sub-basin is, the lower is the CRPSS. The absolute values of the coefficients of MAP $\left(b_{3}\right)$ exhibit an interesting decreasing trend up to day 4 and then an increasing trend from days 6 to 10 . Except day 5 which shows a positive relation between CRPSS and MAP, all other nine days have negative values, suggesting that wetter sub-basins with higher mean annual precipitation tend to have lower CRPSS and the ensemble forecasts are less capable for wetter sub-basins. The degree of negative relationship between 

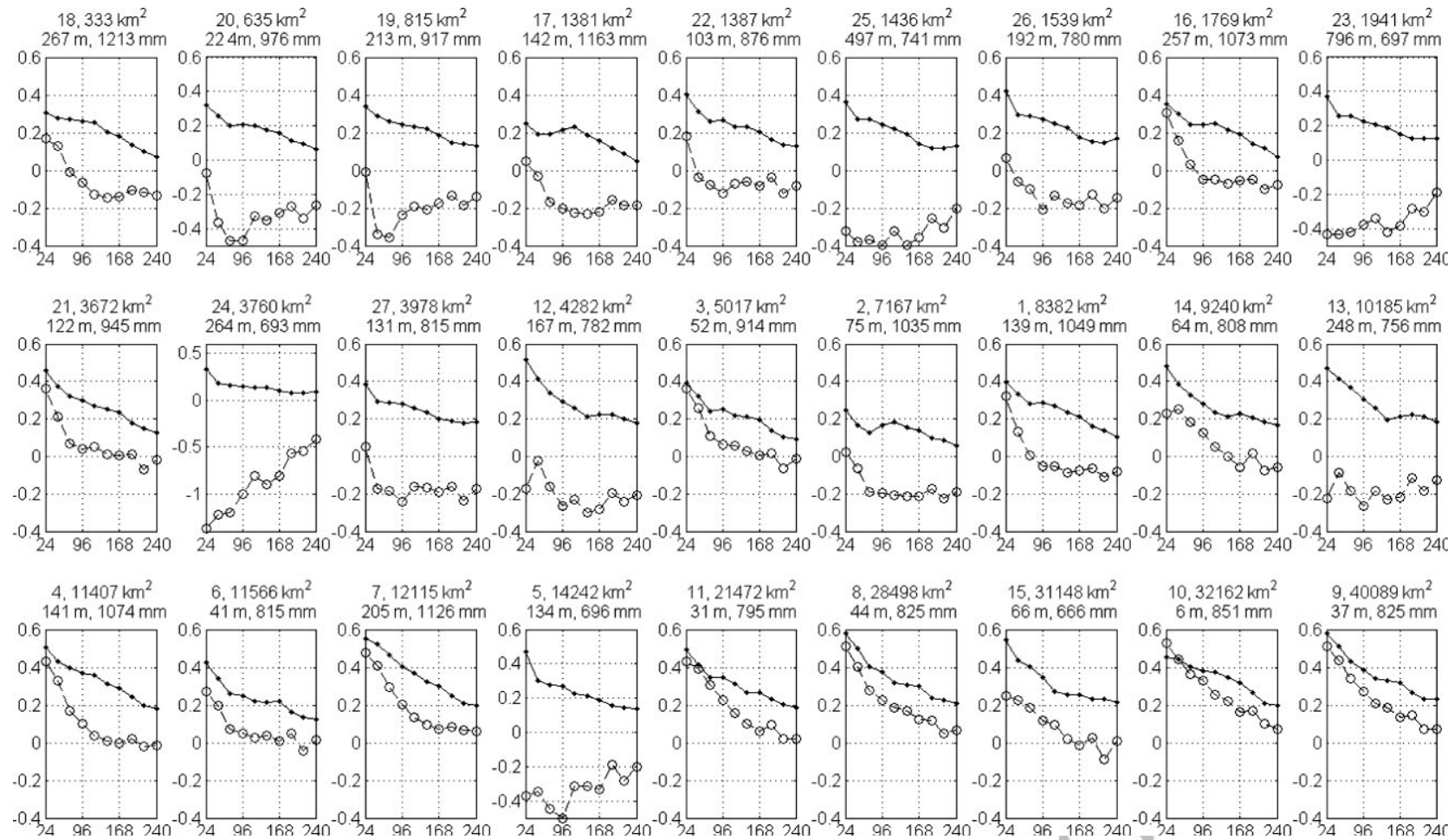

9. $40089 \mathrm{~km}^{2}$ $37 \mathrm{~m}, 825 \mathrm{~mm}$

Figure 4. The mean CRPSS versus lead times for each sub-basin in flooding seasons (line with dots) and non-flooding seasons (line with circles). The score is calculated using $24 \mathrm{~h}$ accumulated precipitation. Sub-plots are presented in ascending order of the sub-basin size. The numbers above each sub-plot are the sub-basin no., size, mean elevation, and mean annual precipitation respectively.

Table 2. The intercepts, coefficients and coefficients of determination $R^{2}$ of the multiple regression of CRPSS for flooding and non-flooding seasons at different lead times.

\begin{tabular}{|c|c|c|c|c|c|}
\hline $\begin{array}{l}\text { Season, } \\
\text { lead time }\end{array}$ & $a$ & $b_{1}$ & $b_{2}$ & $b_{3}$ & $R^{2}$ \\
\hline $\mathrm{F}$, day 1 & 0.636 & 0.161 & -0.034 & -0.348 & 0.756 \\
\hline $\mathrm{F}$, day 2 & 0.400 & 0.212 & -0.009 & -0.159 & 0.740 \\
\hline F, day 3 & 0.342 & 0.192 & 0.046 & -0.154 & 0.720 \\
\hline F, day 4 & 0.285 & 0.152 & 0.012 & -0.066 & 0.719 \\
\hline $\mathrm{F}$, day 5 & 0.164 & 0.140 & 0.020 & 0.067 & 0.655 \\
\hline F, day 6 & 0.209 & 0.124 & -0.002 & -0.011 & 0.682 \\
\hline $\mathrm{F}$, day 7 & 0.201 & 0.130 & -0.046 & -0.013 & 0.819 \\
\hline F, day 8 & 0.218 & 0.111 & -0.034 & -0.088 & 0.818 \\
\hline $\mathrm{F}$, day 9 & 0.237 & 0.102 & -0.007 & -0.150 & 0.823 \\
\hline $\mathrm{F}$, day 10 & 0.319 & 0.090 & -0.002 & -0.278 & 0.834 \\
\hline $\mathrm{nF}$, day 1 & -0.116 & 0.354 & -0.481 & 0.488 & 0.714 \\
\hline $\mathrm{nF}$, day 2 & -0.301 & 0.489 & -0.450 & 0.514 & 0.684 \\
\hline $\mathrm{nF}$, day 3 & -0.159 & 0.464 & -0.366 & 0.184 & 0.693 \\
\hline $\mathrm{nF}$, day 4 & -0.197 & 0.430 & -0.363 & 0.190 & 0.741 \\
\hline $\mathrm{nF}$, day 5 & -0.020 & 0.306 & -0.299 & -0.026 & 0.756 \\
\hline $\mathrm{nF}$, day 6 & -0.081 & 0.273 & -0.358 & 0.053 & 0.763 \\
\hline $\mathrm{nF}$, day 7 & -0.163 & 0.250 & -0.273 & 0.124 & 0.729 \\
\hline $\mathrm{nF}$, day 8 & -0.044 & 0.214 & -0.233 & 0.008 & 0.735 \\
\hline $\mathrm{nF}$, day 9 & -0.256 & 0.238 & -0.180 & 0.175 & 0.687 \\
\hline $\mathrm{nF}$, day 10 & -0.047 & 0.179 & -0.141 & -0.051 & 0.685 \\
\hline
\end{tabular}

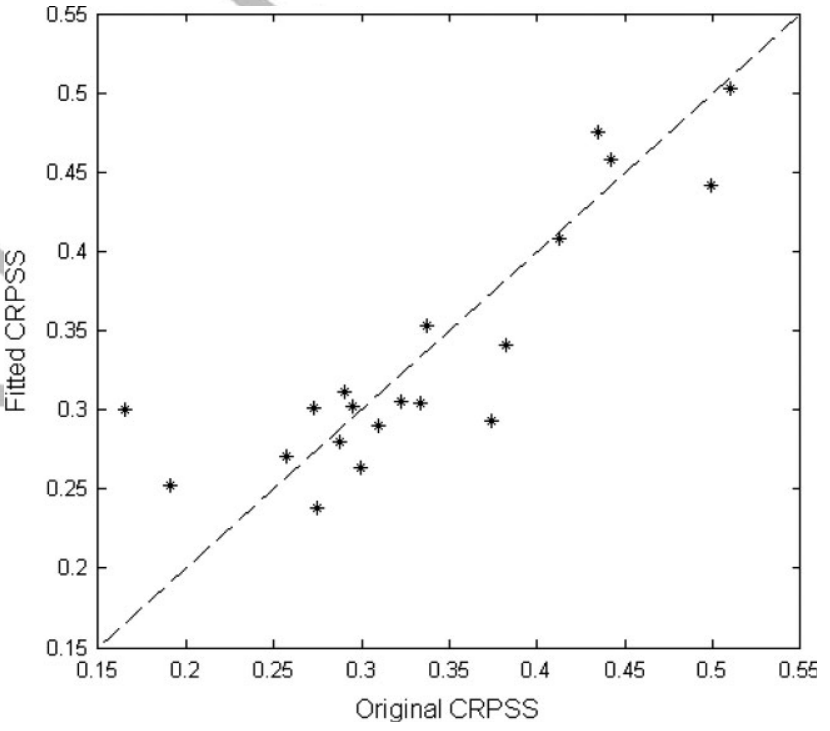

Figure 5. The original and the fitted CRPSS using the multiple regression function flooding season, day 2).

the sub-basin mean elevation when orographic rain events may occur more during the non-flooding seasons. The coefficient $b_{3}$ is mostly positive except on days 5 and 10. It does not show any increasing or decreasing trend as what can be seen in the flooding seasons. The forecast skill tends to be better for those sub-basins with higher mean annual precipitation. It may be easier to forecast rain occurrence and magnitude in a relatively wetter non-flooding season than a drier one.

\subsection{Skill dependency on lead times and aggregation of time steps}

For the non-flooding seasons, the coefficients of determination $R^{2}$ of all ten lead times are generally lower than the ones obtained for the flooding seasons. The coefficient $b_{1}$ is positive for all ten lead times, indicating the forecast skill improves as the subbasin size increases. The coefficient $b_{2}$ is always negative and the absolute values are comparably larger than those in the flooding seasons. This means the forecast skill may be affected more by

The three evaluation scores CRPS, RCRPS and CRPSS were computed at an aggregation of five time steps, namely $3,6,12$, 24 and $48 \mathrm{~h}$, and all available lead times to investigate whether or not the forecast skill depends on the aggregation of time steps and lead times. The obtained scores were then averaged for the five 
flooding and non-flooding seasons respectively. Figure 6 shows the three evaluation scores averaged for (a) the five flooding seasons and (b) the five non-flooding seasons for three selected sub-basins. They are no. 16 (blue dotted lines), 14 (black lines marked with circles) and 10 (red lines marked with crosses). They are randomly selected from each of the three rows of sub-basins shown in Figure 4 to represent three categories of sub-basin sizes. The left, middle and right panels in Figure 6 are CRPS, RCRPS and CRPSS respectively. The rows from top to bottom correspond to five aggregated time steps from 3, 6, 12, 24 to

$1048 \mathrm{~h}$ respectively. Because the ECMWF archives forecasts from

$11 \mathrm{~T}+0 \mathrm{~h}$ to $\mathrm{T}+96 \mathrm{~h}$ at $3 \mathrm{~h}$ intervals, the first row of scores ends 2 at $\mathrm{T}+96 \mathrm{~h}$.
Across the five aggregated time steps, one can see the forecast 1 performance generally deteriorate as the lead times increase, but 2 fluctuate up and down for the 3, 6 and $12 \mathrm{~h}$ time steps. The 3 fluctuating pattern is prominent and seems to follow a periodic 4 cycle every day with an inflexion point occurring after half a day, hence referred to as the diurnal cycle. This particular diurnal cycle will be further discussed in section 4.5 .

It can be observed from the CRPS in Figure 6 that the forecast performance seems to worsen significantly as the time step increases from 3 to $48 \mathrm{~h}$ (values increase from around 0.5 to 5). Because the forecasted variable, precipitation in this study, can assume different magnitudes when it represents different areas, seasons or aggregated time steps, CRPS becomes an
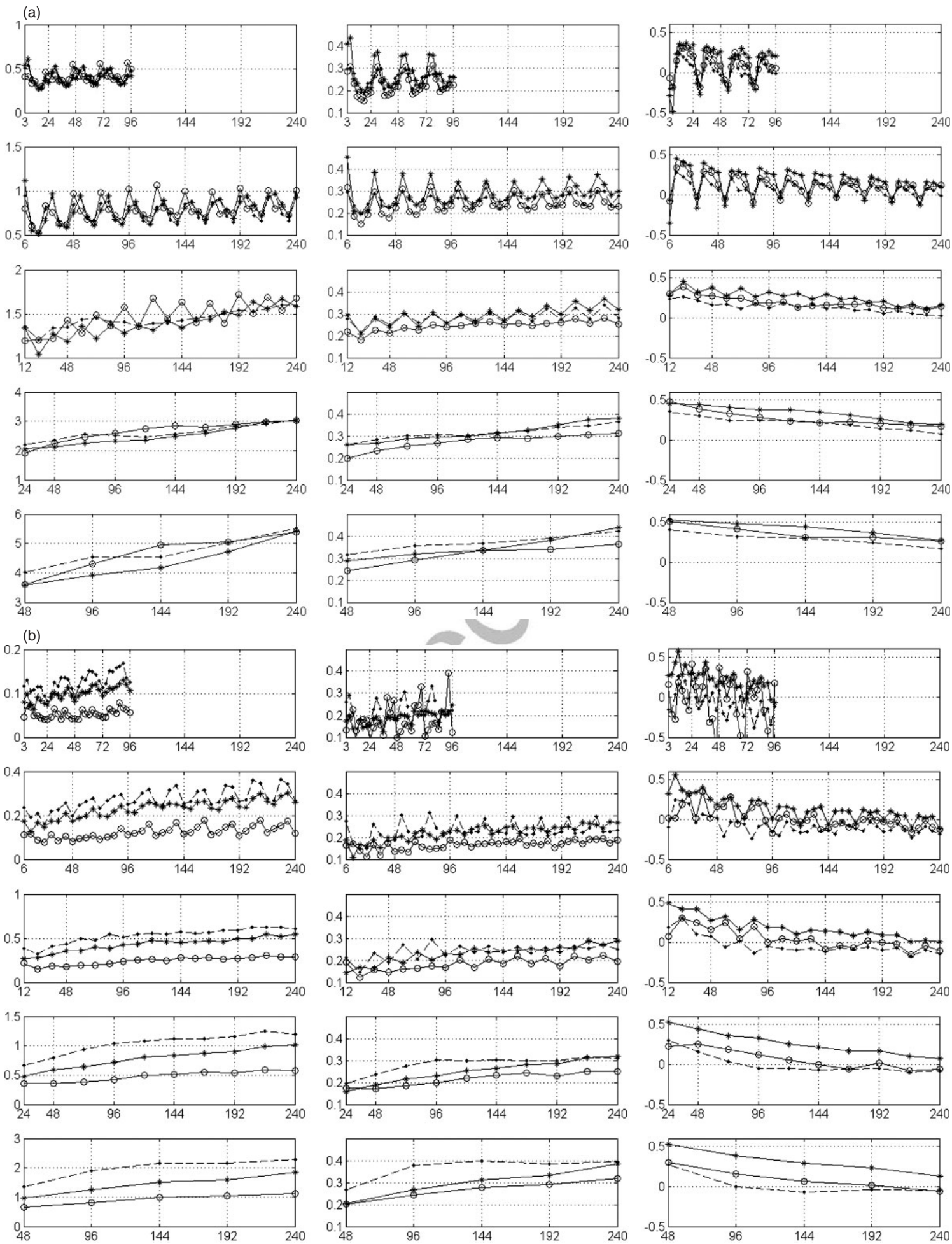

Figure 6. Three performance scores averaged for (a) the five flooding seasons and (b) the five non-flooding seasons for sub-basins nos. 16 (dashed line with dots), 14 (line with circles) and 10 (line with asterisks). Left, middle and right panels are CRPS, RCRPS and CRPSS respectively. The rows from top to bottom correspond to the aggregated time steps of 3, 6, 12, 24 and $48 \mathrm{~h}$ respectively. Because ECMWF archives ensemble forecasts from $\mathrm{T}+0 \mathrm{~h}$ to $\mathrm{T}+96 \mathrm{~h}$ at $3 \mathrm{~h}$ intervals, the first row of scores end at $\mathrm{T}+96 \mathrm{~h}$ 
1 incomparable evaluation score. One needs to use RCRPS or

2 CRPSS if performance comparison needs to be made across different areas, seasons or aggregated time steps. RCPRS is simply a normalised score disregarding the magnitude of the forecasted variable. CRPSS does not only eliminate the effect of the magnitude of the forecasted variable, it also compares the forecasts with the relevant climatology. Therefore, RCRPS and CRPSS are considered in the following comparison. Both RCRPS and CRPSS demonstrate fluctuating patterns for the sub-daily time steps. The scores show evident improvement from 3, 6, 1012 to $24 \mathrm{~h}$ time step. The improvement from 24 to $48 \mathrm{~h}$ is only 11 marginal. This suggests that the forecast performance improves as 12 the aggregation of time steps becomes larger, although marginal 13 once aggregation exceeds $24 \mathrm{~h}$. Sub-basin no. 10 shows the best 14 performance with respect to CRPSS, followed by no. 14, and the worst is no. 16. This reflects the relationship between CRPSS and sub-basin size which has already been discussed in section 4.3. In comparison, Figure 6(b) shows the average scores for the nonflooding seasons. Similar fluctuating patterns can be observed for the sub-daily time steps, but their phases are different from sub-basin to sub-basin.

\subsection{Sub-daily skill}

To study the diurnal cycle in detail, the mean CRPS value of the five flooding and five non-flooding seasons for each of the $27 \mathrm{sub}$ basins at five different aggregated time steps was computed and is shown in Figure 7. ECMWF archives precipitation forecasts at time steps of $\mathrm{T}+0 \mathrm{~h}$ to $\mathrm{T}+96 \mathrm{~h}$ at $3 \mathrm{~h}$ intervals. This is the reason why Figure 7 (a) ends at $96 \mathrm{~h}$ whereas the other four time steps all run up to $240 \mathrm{~h}$. During the flooding seasons, CRPS values in Figure 7(a) of all 27 sub-basins generally increase with the increase of lead times and at a fairly constant pace. In other words, the forecast performance worsens constantly over the lead times, but with the exception of the scores computed at sub-daily time steps. For the $3 \mathrm{~h}$ time step, the CRPS values of most sub-basins decrease from 0300 UTC to a daily minimum at around $1500 \mathrm{UTC}( \pm 3 \mathrm{~h}$ depending on the sub-basin). They then rise to a daily maximum at around Day +10300 UTC (some sub-basins show a small drop between 2100 UTC and 2400 UTC). In other words, the first daily cyclic change in CRPS is a decline from 0300 UTC to 1500 UTC $( \pm 3 \mathrm{~h}$ ) that lasts approximately $12 \mathrm{~h}$, and the second change is a growth from 1500 UTC $( \pm 3 \mathrm{~h})$ to Day +10300 UTC that lasts for another $12 \mathrm{~h}$. These time intervals correspond to the Beijing time 1100-2300 for the decline and 2300-Day +11100 for the growth. The daily minimum CRPS happens at around Beijing time 2300. In terms of the forecast performance, it improves from Beijing time 1100 to 2300 and then drops in the next $12 \mathrm{~h}$. For the non-flooding seasons, the diurnal pattern is not as obvious as for the flooding season. The CRPS values for the $3 \mathrm{~h}$ time interval during the non-flooding seasons seem to fluctuate randomly with a very gentle increasing trend. In Figure 7 (b), the diurnal pattern with the same decline and growth cycles as those of the $3 \mathrm{~h}$ can be clearly seen for the flooding season. For the non-flooding season, the pattern is weak and not consistent for all the sub-basins. In Figure 7(c), the mean CRPS values computed at $12 \mathrm{~h}$ time steps show a different diurnal pattern from those of the 3 and $6 \mathrm{~h}$ time steps. The daily decline starts from 1200 UTC and ends at 2400 UTC, which is followed by a growth from 2400 to 1200 UTC. While the majority of sub-basins show this pattern, there are a number of sub-basins that exhibit a rather opposite pattern. This could be due to the fact that the forecasted precipitation is aggregated for every $12 \mathrm{~h}$ (i.e. $0000-1200,1200-2400$ UTC, etc.) and the aggregation has interfered with the actual dynamics of the rainfall events. Additionally, diurnal cycles vary with the locations of the sub-basins. In Figure 7(d), the forecast performance for both flooding and non-flooding seasons deteriorates rapidly till Day $3(72 \mathrm{~h})$ which is shown as an increase in the mean CRPS values for all the 27 sub-basins. From the 72th to the 240th hour, the rate of increase slows down slightly, especially after the

144th hour (Day 6). In Figure 7(e), the turning point of the mean CRPS values occurs at the 96th hour. Because the precipitation values are aggregated for every $48 \mathrm{~h}$, it is impossible to capture the same turning point as in Figure $7(\mathrm{~d})$.

The diurnal cycle in the observed precipitation for May-September in the domain of central-east China $\left(105-120^{\circ} \mathrm{E}, 26-36^{\circ} \mathrm{N}\right)$ was reported in Yu et al. $(2007 \mathrm{a}, 2007 \mathrm{~b})$. The Huai river basin is located in the upper east of this domain. Their results show that the rainfall events of duration between 1 and $3 \mathrm{~h}$ peak around late afternoon, which may be explained by the diurnal variation of surface solar heating that influences the diurnal variation of low-level atmospheric stability. Rainfall events of duration longer than $6 \mathrm{~h}$ dominate $(>60 \%$ of the total precipitation) in this domain and they tend to peak in the early morning of each day. The reason for this peak is more complex than for the late afternoon one. Nesbitt and Zipser (2003) suggest the nocturnal rain is often caused by mesoscale convective systems (MCS) rather than isolated convection, and the MCS is the strongest after midnight. Chen et al. $(1998,2000)$ and Sun et al. (2005) point out that heavy rainfall of the summer Meiyu front mostly results from well organised MCS overlapping the distinctive stratus cloud. The diurnal pattern of the forecasting performance observed in the flooding season may suggest ECMWF's EPS is weak in capturing the MCS and hence the early morning peaks. There are limited numbers of studies that verify diurnal cycles of NWP-modelled precipitation, although the ability of NWP to capture diurnal precipitation cycles cannot be understated. The diurnal cycles in the mesoscale NWP model from MeteoSwiss are verified using hourly rain-gauge data by Kaufmann et al.(2003). They find the model performs well in winter because there is no diurnal forcing, but fails to reproduce diurnal cycles in summer. The convection starts too early and lasts a very short time in this model and overestimates the amount of precipitation. Guichard et al.(2004) investigate modelling of the diurnal cycle of deep precipitating convection over land using seven single-column models (SCMs) and three cloud-resolving models (CRMs). It was found convection occurs too early in most SCMs due to crude triggering criteria. In the CRMs, the first clouds appear before noon, but surface rainfall is delayed by several hours.

The intra-daily precipitation dynamics were looked at by aggregating both the forecasted and observed precipitation to the multiple of $3 \mathrm{~h}$ from 3 up to $24 \mathrm{~h}$. Figure 8 shows (a) the observed and (b) forecasted mean of the daily aggregated precipitation within each season and for all the 27 sub-basins. In the case of the forecasted mean of the daily aggregated precipitation, it was computed as the mean of the 51 ensemble members for the first eight time steps (Day 1) only. The dark black line is the mean of the mean of the 27 daily aggregated precipitation series. During the non-flooding seasons, there are two major differences between the observed and the forecasted: (i) the spread of the blue lines for the 27 sub-basins is larger in the observed than in the forecasted precipitation; and (ii) the aggregated precipitation values obtained from ensemble forecasts are larger than those from the observed. During the flooding seasons, in addition to the smaller spread in the forecasted daily precipitation, the major contrast lies in the intra-daily precipitation dynamics. The black lines obtained from the observed precipitation are fairly linear, whereas the black lines obtained from the forecasted precipitation show an obvious turning point at the ninth hour in a day. The turning point cannot directly explain the diurnal pattern in the CRPS values obtained for the flooding seasons, but it does indicate that the intra-daily rainfall dynamics are not well simulated by ECMWF's EPS.

\section{Conclusion and outlook}



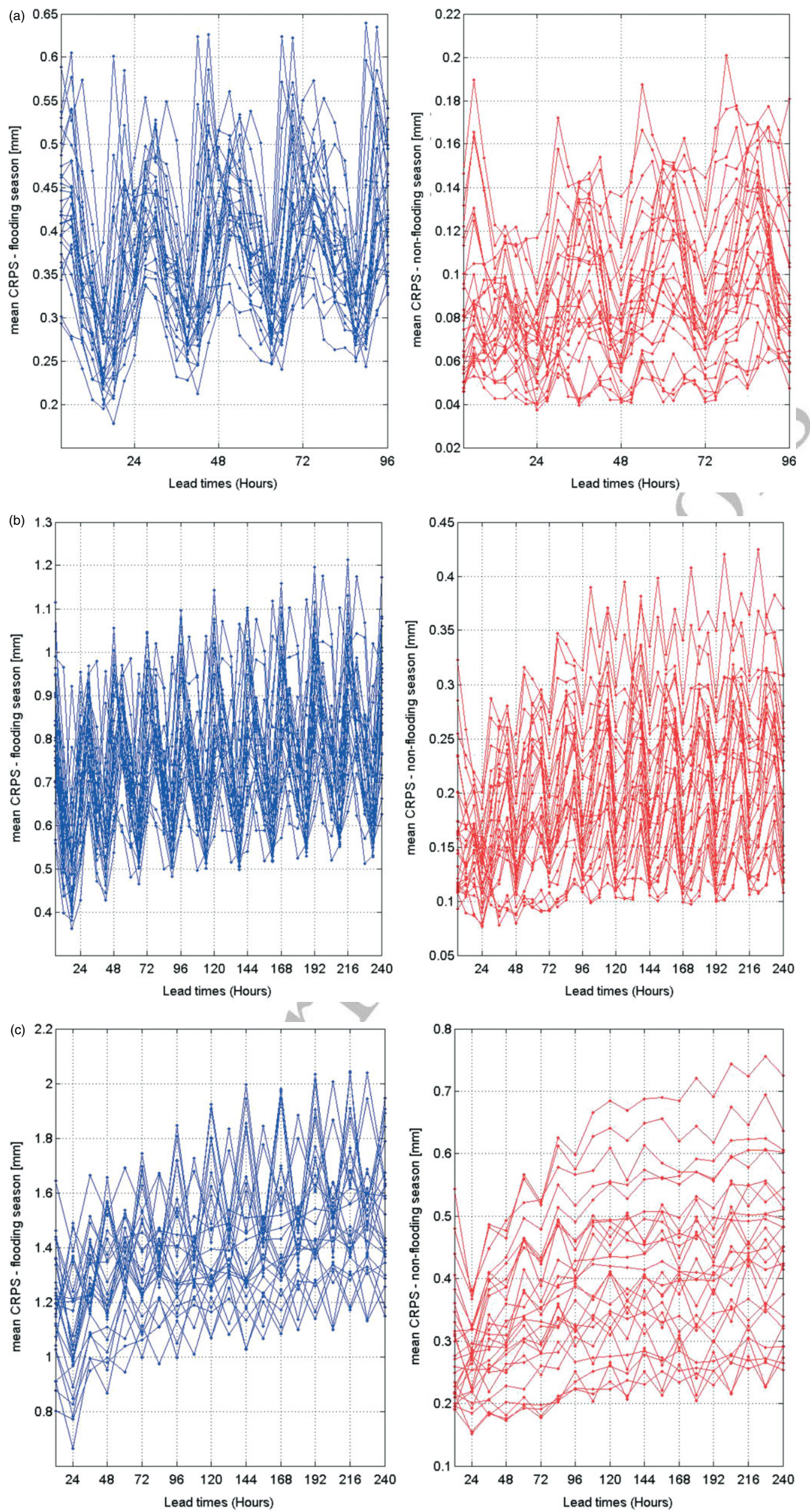

Figure 7. The mean CRPS over five flooding (left panels) and five non-flooding (right panels) seasons for the 27 sub-basins computed at five aggregated time steps: 62 (a) $3 \mathrm{~h}$, (b) $6 \mathrm{~h}$, (c) $12 \mathrm{~h}$, (d) $24 \mathrm{~h}$, and (e) $48 \mathrm{~h}$. This figure is available in colour online at wileyonlinelibrary.com/journal/qj 63 

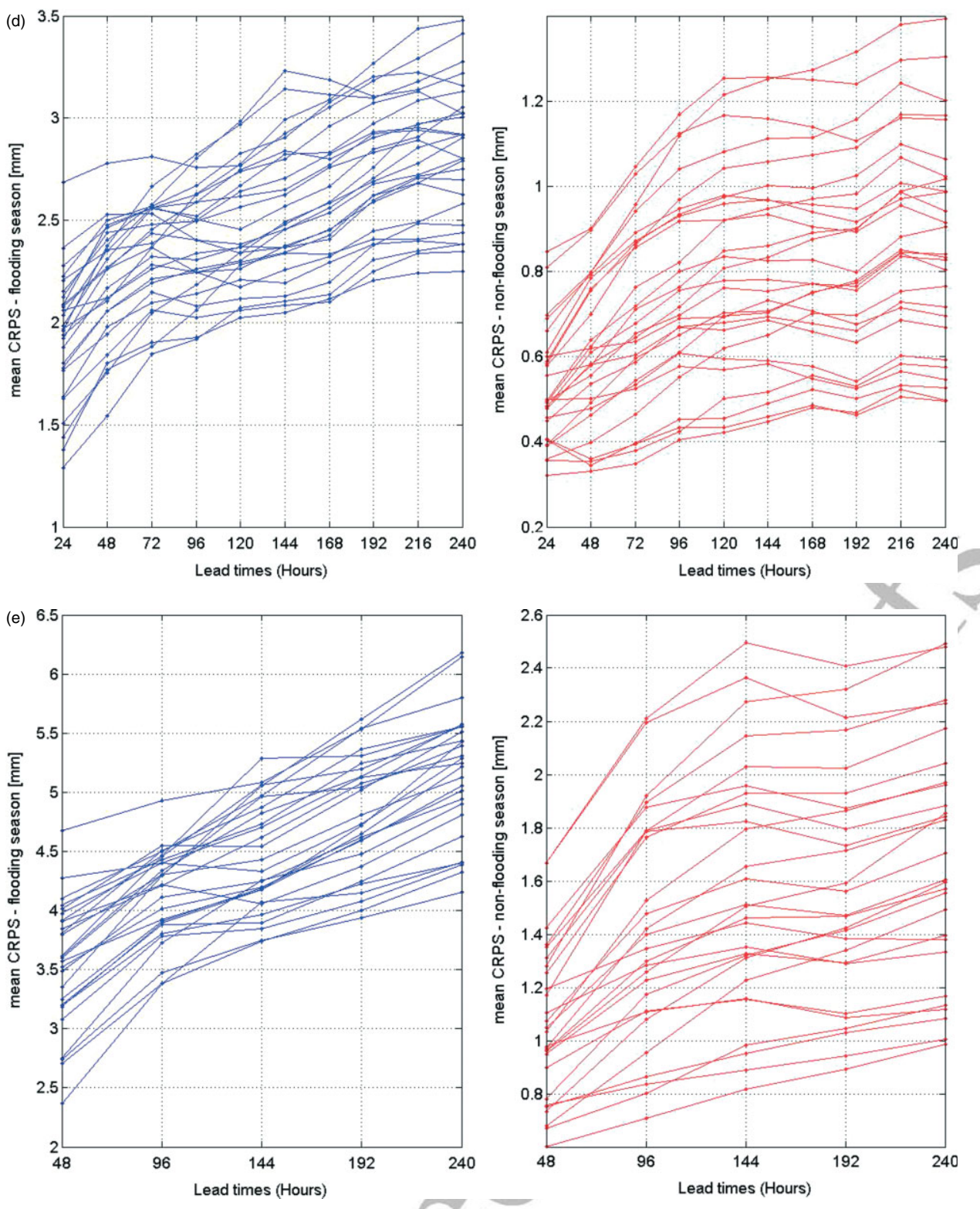

(270 $000 \mathrm{~km}^{2}$ ) representing various geographic and climatic properties. Precipitation forecasts were evaluated in a way relevant to the hydrological processes of runoff, by considering the river basin (and sub-basin) as the spatial units of evaluation as opposed to large aggregated grid-based areas. Only in this way can it be said whether the forecasts have the potential to be useful for hydrological prediction such as flood forecasting. It is strongly recommended that this becomes the norm for precipitation forecast evaluation.

For the observed precipitation, it was found that the CMORPHGauge merged dataset proves to be of good quality when cross-checked with the daily rain-gauge data. The dataset enabled detailed forecast performance evaluation at sub-daily scales for the first time. The dataset covers almost 5 years which allowed a long-term and continuous evaluation, rather than event-based studies. Such continuous evaluation is essential for truly understanding the quality of any forecasting system. The CMORPH-Gauge merged dataset systematically underestimates precipitation, especially high precipitation and this bias should be corrected for different months, seasons and areas to give a more credible evaluation of the forecast performance. This dataset is especially valuable in the assessment of EPS at subdaily scales, as the sub-daily precipitation can be important in hydrological applications (e.g. Wetterhall et al., 2011; Parkes et al., 2013).
Precipitation forecast performance was found to vary with sub-basin properties, aggregated time steps and lead times, and between flooding and non-flooding seasons. This highlights two salient points: forecast performance can only be evaluated effectively if the forecast parameters are understood (lead time, time step) but also importantly if the hydrogeographical attributes of the study area are also considered (e.g. basin elevation, flood seasonality, etc.). The study provides answers to the three scientific questions proposed in the introduction:

(i) For midlatitude sub-basins like the ones in the Huai river basin, ECMWF's ensemble forecasts can be used in forecasting floods with relatively low, medium and high confidence during flooding seasons for sub-basins with sizes $<2000,2000-10000$ and $>10000 \mathrm{~km}^{2}$ respectively. The exception is the sub-basin dominated by high elevations. During non-flooding seasons, no satisfactory skills were found for the sub-basins smaller than $2000 \mathrm{~km}^{2}$ but some reasonable skills for the sub-basins larger than $2000 \mathrm{~km}^{2}$. Overall, the forecasts are more skilful in the flooding seasons than the non-flooding seasons over this basin.

(ii) The forecast skill at each sub-basin depends on the three studied sub-basin hydrogeographical properties of basin size, mean annual precipitation and mean elevation, to various extents, and seasons as well. Because the obtained
41 42 43 44 45 46 47 48 

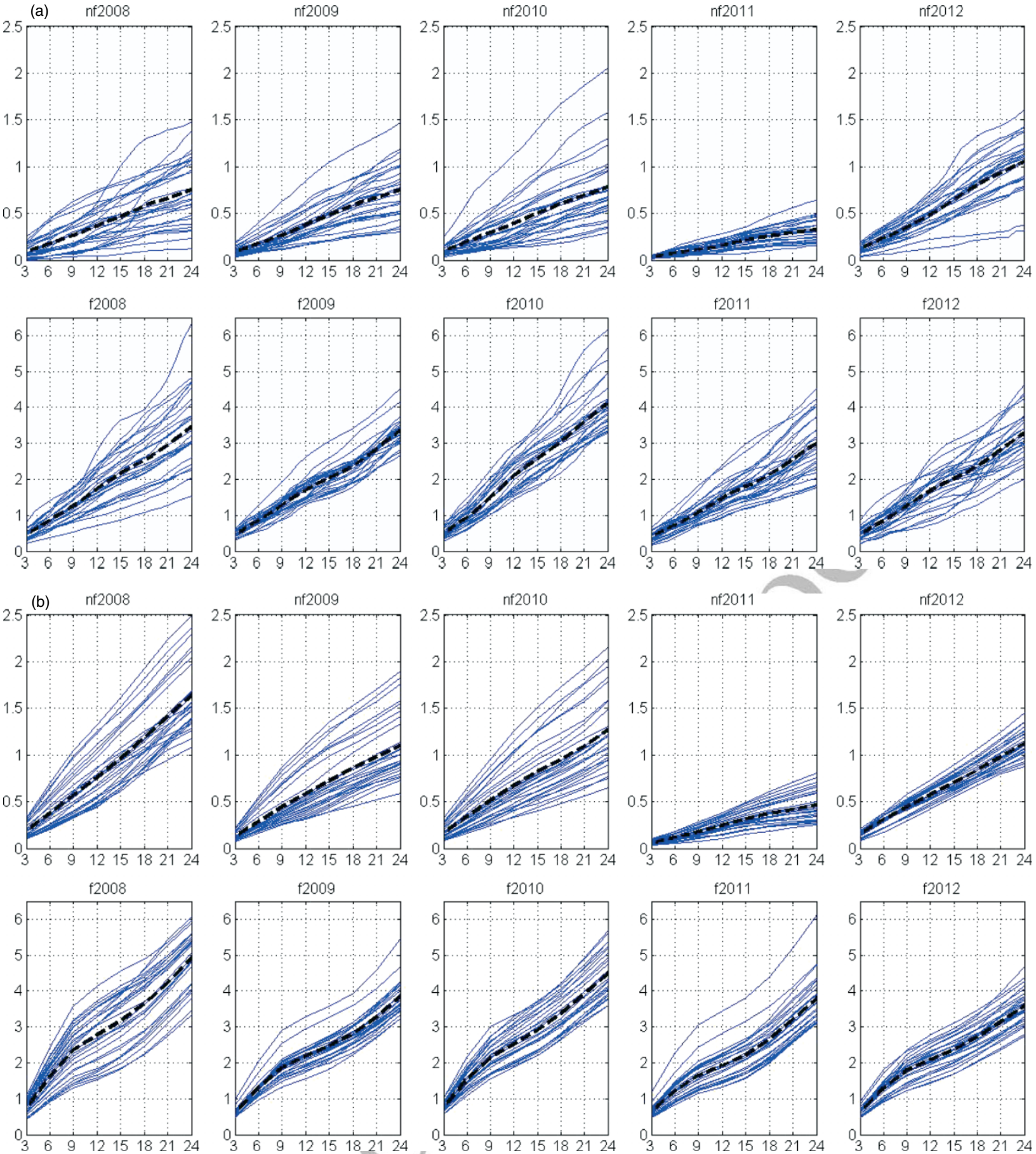

Figure 8. The (a) observed and (b) forecasted mean of the aggregated precipitation within each season and for all the 27 sub-basins. $\mathrm{x}$-axis is the aggregated time in hours. $y$-axis is the precipitation in mm. The 'nf' and 'f above each sub-plot stands for 'non-flooding season' and 'flooding season' respectively. The dashed line is the mean (the 27 sub-basins) of the mean (51 ensemble members) of the daily aggregated precipitation of the 27 sub-basins. This figure is available in colour online at wileyonlinelibrary.com/journal/qj

regression model does not account for the total variability in CRPSS, other variables that can affect the forecast performance may need to be considered. Regardless of the season, larger sub-basins benefit from better forecast skills because the current forecast model is still limited in resolving small-scale events. One needs to be cautious when applying these ensemble forecasts in small-scale sub-basins, especially those smaller than $2000 \mathrm{~km}^{2}$. The higher the sub-basin's mean annual precipitation, the lower the CRPSS. This means the ensemble forecasts are less capable for wetter sub-basins, and in particular for the extreme events. The comparatively lower CRPSS during non-flooding seasons indicate the ensemble forecasts are less skilful in simulating rain occurrence and magnitude in dry seasons. The drier the sub-basin, the more challenges it presents to the model to correctly forecast the rain.
ECMWF's EPS does a fairly satisfactory job in forecasting medium-range precipitation, but needs to improve in forecasting very high or low precipitation. The forecast skill also depends on lead times and aggregation of time steps. Forecast performance worsens as the lead time increases. The forecast performance improves as the time steps are aggregated from $3,6,12$ to $24 \mathrm{~h}$ time step. The improvement from 24 to $48 \mathrm{~h}$ is marginal.

(iii) In flooding seasons, the evaluation scores at sub-daily steps present a prominent and consistent diurnal cycle for all the 27 sub-basins. The forecast performance improves from Beijing time 1100 to 2300 and then drops in the next $12 \mathrm{~h}$. In non-flooding seasons, the diurnal cycle also exists at sub-daily time steps, although not consistently across the 27 sub-basins. The reasons for the diurnal cycle in observed precipitation are still not well understood.
2

3

4

9

10

11

12

13

14

15

16

17

18

19

20

21

22

23

24

25

26

27

28

29

30

31

32

33

34

35

36

37

38

39

40

41

42 
The result suggests ECMWF's ensemble forecasts may be unsuccessful in capturing the nocturnal cycle and the MCS in the study domain. The result also shows the intra-daily rainfall dynamics are not well simulated by ECMWF's EPS and hence the sub-daily ensemble forecasts generated by the current model version do not benefit the forecasters. The model performance at sub-daily steps and reasons for failures need to be studied in more detail in the future.

The Huai river basin used in this study was selected because it is representative of many types of river basins around the world and thus conclusions can be to some extent generalised. It can be said that ECMWF EPS precipitation forecasts are generally skilful for flood forecasting especially in large river sub-basins. However, future research is encouraged in other areas of the world and even at the global scale (Alfieri et al., 2013). Future research could also consider in more detail the impact of postprocessing methods (Schaake et al., 2010) and the nature of the rainfall patterns and intensities during flood seasons. As the use of EPS forecasts in flood forecasting becomes more widespread across the globe, studies of this nature will become increasingly important in providing benchmarks for operational forecasting. Evaluating precipitation forecasts in a hydrologically relevant way, as demonstrated in this study, is essential in order to fully understand forecast performance.

\section{Acknowledgements}

This study was partially financed by the UK ESRC research project 'Improving the communication and use of ensemble flood predictions' (RES-189-25-0286) and partially supported by the National Natural Science Foundation of China (41130639 and 51179045) and China Meteorological Administration Special Public Welfare Research Fund (GYHY201006037). The observed precipitation data were provided by the Climatic Data Centre, National Meteorological Information Centre, China Meteorological Administration. We thank the two anonymous reviewers for their careful revision, which helped improve this manuscript.

\section{References}

Alfieri L, Salamon P, Pappenberger F, Wetterhall F, Thielen J. 2012. Operational early warning systems for water-related hazards in Europe. Environ. Sci. Policy 21: 35-49, DOI: 10.1016/i.envsci.2012.01.008.

Alfieri L, Burek P, Dutra E, Krzeminski B, Muraro D, Thielen J, Pappenberger F. 2013. GloFAS - Global ensemble streamflow forecasting and flood early warning. Hydrol. Earth Syst. Sci. 17: 1161-1175, DOI: 10.5194/hess-171161-2013.

Bao C-L. 1987. Synoptic Meteorology in China. Springer-Verlag: Berlin.

Bougeault P, Toth Z, Bishop C, Brown B, Burridge D, Chen D, Ebert E, Fuentes M, Hamill T, Mylne KR, Nicolau J, Paccagnella T, Park Y-Y, Parsons D, Raoult B, Schuster D, Silva Dias P, Swinbank R, Takeuchi Y, Tennant W, Wilson L, Worley S. 2010. The THORPEX Interactive Grand Global Ensemble (TIGGE). Bull. Am. Meteorol. Soc. 91: 1059-1072.

Bröcker J. 2012. Evaluating raw ensembles with the continuous ranked probability score. Q. J. R. Meteorol. Soc. 138: 1611-1617, DOI: 10.1002/ qj.1891.

Brown TA. 1974. Admissible Scoring Systems for Continuous Distributions, Manuscript P-5235. The Rand Corporation: Santa Monica, CA. Available from The Rand Corporation, 1700 Main St. Santa Monica, CA 90407-2138. Buizza R. 2008. The value of probabilistic prediction. Atmos. Sci. Lett. 9: 36-42, DOI: $10.1002 /$ asl.170.

Chen S-J, Kuo Y-H, Wang W, Tao Z-Y, Cui B. 1998. A modeling case study of heavy rainstorms along the Meiyu front. Mon. Weather Rev. 126: 2330-2351, DOI: 10.1175/1520-0493(1998)126<2330:AMCSOH > 2.0.CO;2.

Chen S-J, Wang W, Lau K-H, Zhang Q-H, Chung Y-S. 2000. Mesoscale convective systems along the Meiyu front in a numerical model. Meteorol. Atmos. Phys. 75: 149-160.

Cheng H-Q. 2004. 'Spatial variation, causes and forecasting models of storm events in the Huai River basin', Masters thesis, Chinese Academy of Meteorological Sciences. Unpublished (in Chinese).

Cloke HL, Pappenberger F. 2008. Evaluating forecasts of extreme events for hydrological applications: An approach for screening unfamiliar performance measures. Meteorol. Appl. 15: 181-197.

Cloke HL, Pappenberger F. 2009. Ensemble flood forecasting: A review. J. Hydrol. 375: 613-626.
Demeritt D, Nobert S, Cloke HL, Pappenberger F. 2010. Challenges in communicating and using ensembles in operational flood forecasting. Meteorol. Appl. 17: 209-222, DOI: 10.1002/met.194.

De Roo A, Thielen J, Salamon P, Bogner K, Nobert S, Cloke HL, Demeritt D, Younis J, Kalas M, Bodis K, Muraro D, Pappenberger F. 2011. Quality control, validation and user feedback of the European Flood Alert System (EFAS). Int. J. Digital Earth 4:(Suppl. 1): 77-90.

Fu A. 1991. A Brief Introduction of River System in Hongruhe River Basin. Henan Province Prospecting and Designing Institute of Water Conservancy: Zhengzhou. Unpublished.

Guichard F, Petch JC, Redelsperger J-L, Bechtold P, Chaboureau J-P, Cheinet S, Grabowski W, Grenier H, Jones CG, Köhler M, Piriou J-M, Tailleux R, Tomasini M. 2004. Modelling the diurnal cycle of deep precipitating convection over land with cloud-resolving models and single-column models. Q. J. R. Meteorol. Soc. 130: 3139-3172, DOI: 10.1256/qj.03.145.

Hamill TM, Mullen SL, Snyder C, Baumhefner DP, Toth Z. 2000. Ensemble forecasting in the short to medium range: Report from a workshop. Bull. Am. Meteorol. Soc. 81: 2653-2664, DOI: 10.1175/1520-0477 (2000)081<2653:EFITST > 2.3.CO;2.

He Y, Wetterhall F, Cloke HL, Pappenberger F, Wilson M, Freer J, McGregor G 2009. Tracking the uncertainty in flood alerts driven by grand ensemble weather predictions. Meteorol. Appl. 16: 91-101, DOI: 10.1002/met.132.

He Y, Wetterhall F, Bao H-J Cloke HL, Li Z-J, Pappenberger F, Hu Y-Z, Manful D, Huang Y-C. 2010. Ensemble forecasting using TIGGE for the July-September 2008 floods in the Upper Huai sub-basin - A case study. Atmos. Sci. Lett. 11: 132-138, DOI: 10.1002/asl.270.

Hersbach H. 2000. Decomposition of the continuous ranked probability score for ensemble prediction systems. Weather and Forecasting 15: 559-570, DOI: 10.1175/1520-0434(2000)015<0559:DOTCRP > 2.0.CO;2.

Huai River Commission, Ministry of Water Resources. 1999. Atlas of Huai River Basin. Science Press: Beijing.

Huai River Commission, Ministry of Water Resources. 2010. Huai River Storms and Floods in 2007. China Water Power Press: Beijing (in Chinese).

Joyce RJ, Janowiak JE, Arkin PA, Xie P. 2004. CMORPH: A method that produces global precipitation estimates from passive microwave and infrared data at high spatial and temporal resolution. J. Hydrometeorol. 5: 487-503.

Kaufmann P, Schubiger F, Binder P. 2003. Precipitation forecasting by a mesoscale numerical weather prediction (NWP) model: Eight years of experience. Hydrol. Earth Syst. Sci. 7: 812-832, DOI: 10.5194/hess-7-8122003

Leith CE. 1974. Theoretical skill of Monte Carlo forecasts. Mon. Weather. Rev. 102: 409-418, DOI: 10.1175/1520-0493(1974)102<0409:TSOMCF> 2.0.CO;2.

Liu Y, Duan Q, Zhao L, Ye A, Tao Y, Miao C, Mu X, Schaake JC. 2013. Evaluating the predictive skill of post-processed NCEP GFS ensemble precipitation forecasts in China's Huai river basin. Hydrol. Process. 27: 57-74, DOI: 10.1002/hyp.9496.

Lorenz EN. 1963. Deterministic nonperiodic flow. J. Atmos. Sci. 20: 130-141. Lorenz EN. 1969. The predictability of a flow which possesses many scales of motion. Tellus 21: 289-307.

Matheson JE, Winkler RL. 1976. Scoring rules for continuous probability distributions. Manage. Sci. 22: 1087-1095.

Molteni F, Buizza R, Palmer TN, Petroliagis T. 1996. The ECMWF Ensemble Prediction System: Methodology and validation. Q. J. R. Meteorol. Soc. 122: 73-119, DOI: 10.1002/qj.49712252905.

Nesbitt SW, Zipser EJ. 2003. The diurnal cycle of rainfall and convective intensity according to three years of TRMM measurements. J. Climate 16: $1456-1475$.

Ning Y, Qian M, Wang Y-T. 2003. Huai River Basin Hydraulic Handbook. Science Press: Beijing (in Chinese).

Pan Y, Shen Y, Yu JJ, Zhao P. 2012. Analysis of the combined gauge-satellite hourly precipitation over China based on the OI technique. Acta Meteorol. Sin. 70: 1381-1389 (in Chinese with an English abstract).

Pappenberger F, Buizza R. 2009. The skill of ECMWF predictions for hydrological modelling. Weather and Forecasting 24: 749-766.

Pappenberger F, Beven KJ, Hunter N, Gouweleeuw B, Bates P, de Roo A, Thielen J. 2005. Cascading model uncertainty from medium range weather forecasts (10 days) through a rainfall-runoff model to flood inundation predictions within the European Flood Forecasting System (EFFS). Hydrol. Earth Syst. Sci. 9: 381-393.

Pappenberger F, Scipal K, Buizza R. 2008a. Hydrological aspects of meteorological verification. Atmos. Sci. Lett. 9: 43-52.

Pappenberger F, Bartholmes J, Thielen J, Cloke HL, de Roo A, Buizza R. 2008b. New dimensions in early flood warning across the globe using grandensemble weather predictions. Geophys. Res. Lett. 35: L10404, DOI: 10.1029/ 2008 GL033837.

Pappenberger F, Bogner K, Wetterhall F, He Y, Cloke HL, Thielen J. 2011a. Forecast convergence score: A forecaster's approach to analysing hydro-meteorological forecast systems. Adv. Geosci. 29: 27-32, DOI: 10.5194/adgeo-29-27-2011.

Pappenberger F, Thielen J, del Medico M. 2011b. The impact of weather forecast improvements on large scale hydrology: Analysing a decade of forecasts of the European Flood Alert System. Hydrol. Process. 25: DOI: 10.1002/hyp.7772, 2010.

Park Y-Y, Buizza R, Leutbecher M. 2008. TIGGE: Preliminary results on comparing and combining ensembles. Q. J. R. Meteorol. Soc. 134: 2029-2050, DOI: $10.1002 / q j .334$.

Parkes BL, Wetterhall F, Pappenberger F, He Y, Malamud BD, Cloke HL. 2013. Assessment of a 1-hour gridded precipitation dataset to drive a hydrological 3 4 5

6 10 
model: A case study of the summer 2007 floods in the Upper Severn, UK. Hydrol. Res. 44: 89-105, DOI: 10.2166/nh.2011.025.

Schaake J, Pailleux J, Arritt R, Hamill T, Luo L, Martin E, McCollor D, Pappenberger F. 2010. Summary of recommendations of the first workshop on Postprocessing and Downscaling Atmospheric Forecasts for Hydrologic Applications held at Météo-France, Toulouse, France, 15-18 June 2009. Atmos. Sci. Lett. 11: 59-63.

Shen Y, Pan Y, Yu JJ, Zhao P. 2013. Quality assessment of hourly merged precipitation products over China. Trans. Atmos. Sci. 36: 37-46 (in Chinese with an English abstract).

Sun J-H, Zhang X-L, Qi L-L, Zhao S-X. 2005. An analysis of a meso-ß system in a Mei-yu front using the intensive observation data during CHeRES 2002. Adv. Atmos. Sci. 22: 278-289.

Svensson C, Rakhecha PR. 1998. Estimation of probable maximum precipitation for dams in the Hongru river sub-basin. China Theor. Appl. Climatol. 59: 79-91.

Thielen J, Schaake J, Hartman R, Buizza R. 2008. Aims, challenges and progress of the Hydrological Ensemble Prediction Experiment (HEPEX) following the third HEPEX workshop held in Stresa 27 to 29 June 2007. Atmos. Sci. Lett. 9: 29-35, DOI: 10.1002/asl.168.

Thirel G, Rousset-Regimbeau F, Martin E, Habets F. 2008. On the impact of short-range meteorological forecasts for ensemble streamflow predictions. J. Hydrometeorol. 9: 1301-1317.

Trinh BN, Thielen-del Pozo J, Thirel G. 2013. The reduction continuous rank probability score for evaluating discharge forecasts from hydrological ensemble prediction systems. Atmos. Sci. Lett. 14: 61-65, DOI: 10.1002/ 1 asl2.417.

Velázquez JA, Petit T, Lavoie A, Boucher M-A, Turcotte R, Fortin V, Anctil F. 2009. An evaluation of the Canadian global meteorological ensemble prediction system for short-term hydrological forecasting. Hydrol. Earth 4 Syst. Sci. 13: 2221-2231, DOI: 10.5194/hess-13-2221-2009.

Voisin N, Pappenberger F, Lettenmaier DP, Buizza R, Schaake JC. 5

2011. Application of a medium-range global hydrologic probabilistic 6 forecast scheme to the Ohio River basin. Weather and Forecasting 26: 7 425-446.

Wetterhall F, He Y, Cloke H, Pappenberger F. 2011. Effects of temporal resolution of input precipitation on the performance of hydrological forecasting. Adv. Geosci. 29: 21-25, DOI: 10.5194/adgeo-29-21-2011.

Xie P-P, Chen M-Y, Yang S, Yatagai A, Hayasaka T, Fukushima Y, Liu CM. 2007. A gauge-based analysis of daily precipitation over East Asia. J. Hydrometeorol. 8: 607-626, DOI: 10.1175/JHM583.1.

Yang C, Yan Z-W, Shao Y-H. 2012. Probabilistic precipitation forecasting based on ensemble output using generalized additive models and Bayesian model averaging. Acta Meteorol. Sin. 26: 1-12.

Yu R-C, Xu Y-P, Zhou T-J, Li J. 2007a. Relation between rainfall duration and diurnal variation in the warm season precipitation over central eastern China. Geophys. Res. Lett. 34: L13703, DOI: 10.1029/2007GL030315.

Yu R-C, Zhou T-J, Xiong A-Y, Zhu Y-J, Li J-M. 2007b. Diurnal variations of summer precipitation over contiguous China. Geophys. Res. Lett. 34: L01704, DOI: $10.1029 / 2006 \mathrm{GL} 028129$.

\section{8} .

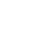

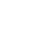
7 9 


\section{Queries from the Copyeditor:}

AQ1 Please provide the department name for affiliations ' $a, c$, g'.

AQ2 Please provide the details for the corresponding author.

AQ3 Buizza, 1999 has not been included in the Reference List, please supply full publication details.

AQ4 We have changed the citation from "Huai River Commission" to "Huai River Commission, Ministry of Water Resources". Please check if it is fine.

AQ5 The publication year of "2012" has been changed "2013" to match the publication year given in the list. Please confirm that this is correct.

AQ6 Figures 2-8 are poor quality. Kindly resupply.

AQ7 Please provide the significance of italic values in Table 2.

AQ8 In the caption of Figure 6, this was mentioned as asterisks. Please check.

AQ9 Please indicate the colors in the caption that was used in the artwork of Figure 7.

AQ10 Please indicate the colors in the caption that was used in the artwork of Figure 8.

AQ11 Please provide the city location of university for Reference Cheng, 2004.

AQ12 Please clarify if this article has since been published for Reference Cheng, 2004.

AQ13 Please clarify if this article has since been published for Reference Fu, 1991.

AQ14 Please provide the page range for Reference Pappenberger et al. $2011 \mathrm{~b}$.

AQ15 Park et al.2008 has not been cited in the text. Please indicate where it should be cited; or delete from the Reference List. 
Required software to e-Annotate PDFs: Adobe Acrobat Professional or Adobe Reader (version 7.0 or above). (Note that this document uses screenshots from Adobe Reader $\mathbf{X}$ )

The latest version of Acrobat Reader can be downloaded for free at: http://get.adobe.com/uk/readerl

Once you have Acrobat Reader open on your computer, click on the Comment tab at the right of the toolbar:

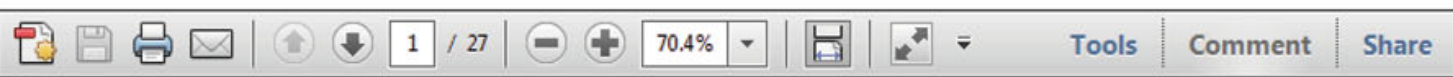

This will open up a panel down the right side of the document. The majority of tools you will use for annotating your proof will be in the Annotations section, pictured opposite. We've picked out some of these tools below:

\begin{tabular}{|l|l|}
\hline Annotations \\
\hline $\mathrm{T}_{\Delta} \mathrm{T}_{\Delta} \mathrm{T}$ & $\mathrm{T}$ \\
\hline
\end{tabular}

1. Replace (Ins) Tool - for replacing text.

Strikes a line through text and opens up a text box where replacement text can be entered.

How to use it

- Highlight a word or sentence.

- Click on the Replace (Ins) icon in the Annotations section.

- Type the replacement text into the blue box that appears.

Idard tramework for the analysis of $\mathrm{m}$ icy Nevertheless, it alse-led to exog، ole of strateg . . . . . . $\mathrm{f}$. $\begin{array}{ll} & \text { aber of comp } \\ \text { 08/06/2011 15:58:17 }\end{array}$ - o is that the $\mathbf{s} 1$, which led of nain compo $\quad$ bc level, are exe nc


M henreforth) 1 we onen the 'hlack t

3. Add note to text Tool - for highlighting a section to be changed to bold or italic.

T. Highlights text in yellow and opens up a text box where comments can be entered.

How to use it

- Highlight the relevant section of text.

- Click on the Add note to text icon in the Annotations section.

- Type instruction on what should be changed regarding the text into the yellow box that appears.

namic responses of mark ups ent with the VAR evidence

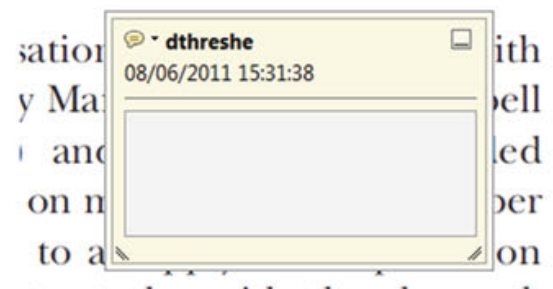

etent ales with the demand.
2. Strikethrough (Del) Tool - for deleting text.

Thes a red line through text that is to be deleted.

How to use it

- Highlight a word or sentence.

- Click on the Strikethrough (Del) icon in the Annotations section.

there is no room for extra prohts al : ups are zero and the number of set) values are not determined by Blanchard and Kiyotaki (1987), rfect competition in general equilil ts of aggregate demand and supply lassical framework assuming monol a on evorensuc numher of firms

4. Add sticky note Tool - for making notes at specific points in the text.

Marks a point in the proof where a comment needs to be highlighted.

How to use it

- Click on the Add sticky note icon in the Annotations section.

- Click at the point in the proof where the comment should be inserted.

- Type the comment into the yellow box that appears.

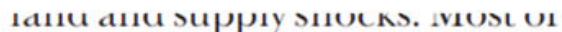

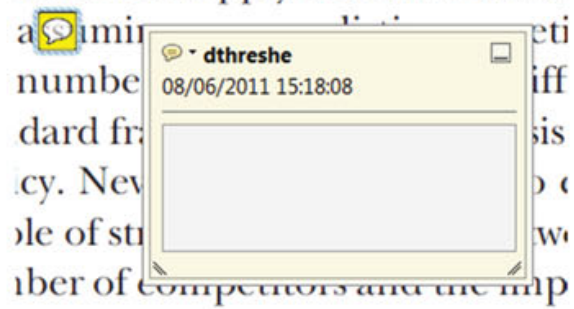
is that the structure of the sects 
5. Attach File Tool - for inserting large amounts of text or replacement figures.

Inserts an icon linking to the attached file in the appropriate pace in the text.

\section{How to use it}

- Click on the Attach File icon in the Annotations section.

- Click on the proof to where you'd like the attached file to be linked.

- Select the file to be attached from your computer or network.

- Select the colour and type of icon that will appear in the proof. Click OK.

E N D

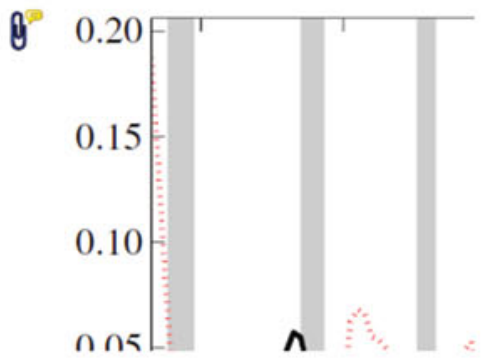

6. Add stamp Tool - for approving a proof if no corrections are required.

- Inserts a selected stamp onto an appropriate place in the proof.

\section{How to use it}

- Click on the Add stamp icon in the Annotations section.

- Select the stamp you want to use. (The Approved stamp is usually available directly in the menu that appears).

- Click on the proof where you'd like the stamp to appear. (Where a proof is to be approved as it is, this would normally be on the first page).

If the Dusiness cycie, starting with the on perfect competition, constant ret

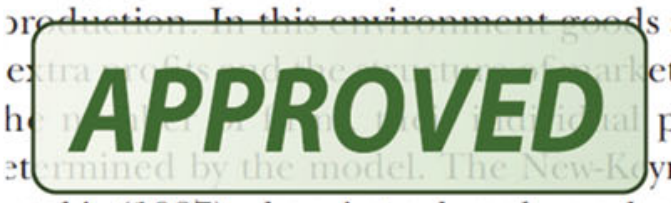
otaki (1987), has introduced produc general equilibrium models with nomin:

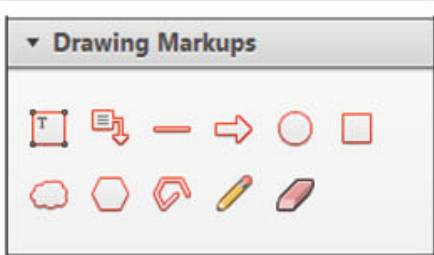

\section{How to use it}

- Click on one of the shapes in the Drawing Markups section.

- Click on the proof at the relevant point and draw the selected shape with the cursor.

- To add a comment to the drawn shape, move the cursor over the shape until an arrowhead appears.

- Double click on the shape and type any text in the red box that appears.
7. Drawing Markups Tools - for drawing shapes, lines and freeform annotations on proofs and commenting on these marks.

Allows shapes, lines and freeform annotations to be drawn on proofs and for comment to be made on these marks..

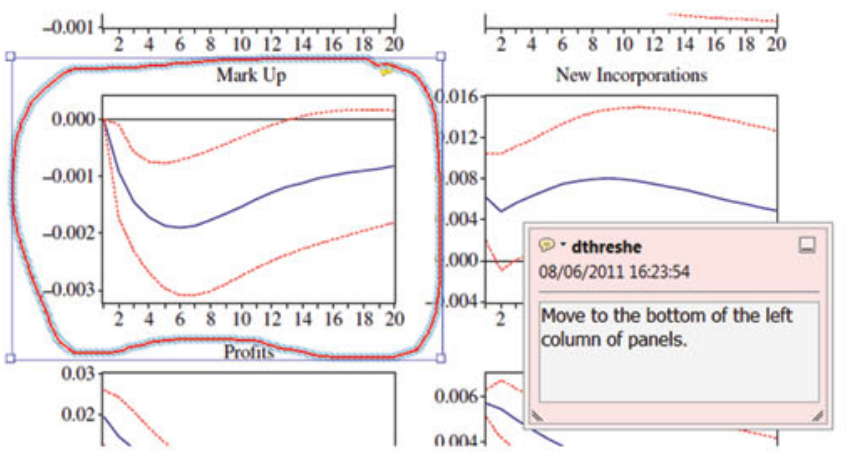

For further information on how to annotate proofs, click on the Help menu to reveal a list of further options:

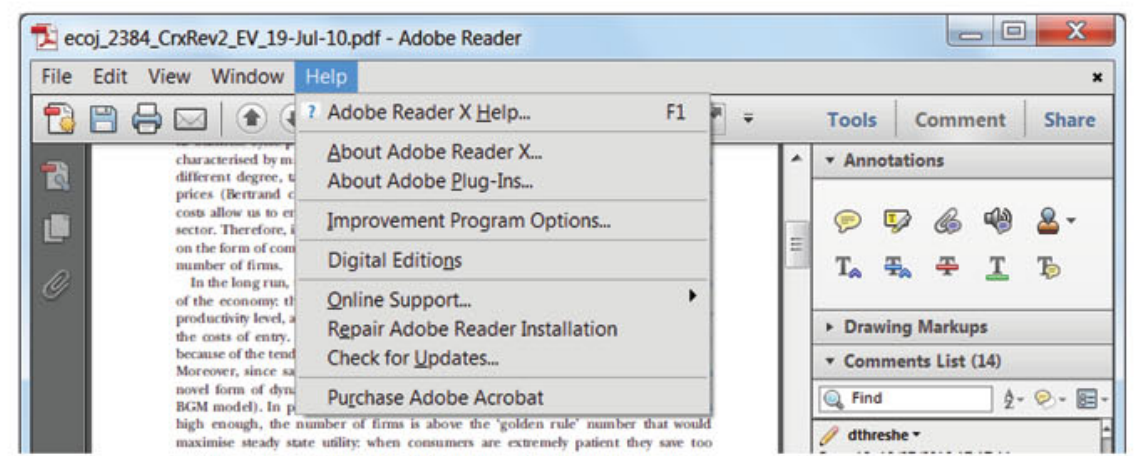

